\title{
Dusty wind of W Hydrae
}

\section{Multi-wavelength modelling of the present-day and recent mass loss}

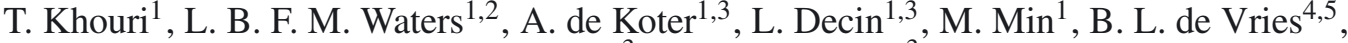 \\ R. Lombaert ${ }^{3}$, and N. L. J. Cox ${ }^{3}$ \\ 1 Astronomical Institute "Anton Pannekoek", University of Amsterdam, PO Box 94249, 1090 GE Amsterdam, The Netherlands \\ e-mail: theokhouri@gmail.com \\ 2 SRON Netherlands Institute for Space Research, Sorbonnelaan 2, 3584 CA Utrecht, The Netherlands \\ 3 Instituut voor Sterrenkunde, KU Leuven, Celestijnenlaan 200D B-2401, 3001 Leuven, Belgium \\ 4 AlbaNova University Centre, Stockholm University, Department of Astronomy, 10691 Stockholm, Sweden \\ 5 Stockholm University Astrobiology Centre, 10691 Stockholm, Sweden
}

Received 1 October 2014 / Accepted 11 March 2015

\begin{abstract}
Context. Low- and intermediate-mass stars go through a period of intense mass-loss at the end of their lives, during the asymptotic giant branch (AGB) phase. While on the AGB a significant part, or even most, of their initial mass is expelled in a stellar wind. This process controls the final stages of the evolution of these stars and contributes to the chemical evolution of galaxies. However, the wind-driving mechanism of AGB stars is not yet well understood, especially so for oxygen-rich sources. Characterizing both the present-day mass-loss rate and wind structure and the evolution of the mass-loss rate of such stars is paramount to advancing our understanding of this processes.

Aims. We study the dusty wind of the oxygen-rich AGB star W Hya to understand its composition and structure and shed light on the mass-loss mechanism.

Methods. We modelled the dust envelope of W Hya using an advanced radiative transfer code. We analysed our dust model in the light of a previously calculated gas-phase wind model and compared it with measurements available in the literature, such as infrared spectra, infrared images, and optical scattered light fractions.

Results. We find that the dust spectrum of W Hya can partly be explained by a gravitationally bound dust shell that probably is responsible for most of the amorphous $\mathrm{Al}_{2} \mathrm{O}_{3}$ emission. The composition of the large $(\sim 0.3 \mu \mathrm{m})$ grains needed to explain the scattered light cannot be constrained, but probably is dominated by silicates. Silicate emission in the thermal infrared was found to originate from beyond $40 \mathrm{AU}$ from the star. In our model, the silicates need to have substantial near-infrared opacities to be visible at such large distances. The increase in near-infrared opacity of the dust at these distances roughly coincides with a sudden increase in expansion velocity as deduced from the gas-phase CO lines. The dust envelope of W Hya probably contains an important amount of calcium but we were not able to obtain a dust model that reproduces the observed emission while respecting the limit set by the gas mass-loss rate. Finally, the recent mass loss of W Hya is confirmed to be highly variable and we identify a strong peak in the mass-loss rate that occurred about 3500 years ago and lasted for a few hundred years.
\end{abstract}

Key words. scattering - stars: AGB and post-AGB - stars: individual: W Hydrae - stars: winds, outflows - radiative transfer solid state: refractory

\section{Introduction}

Low- and intermediate-mass stars in the last stages of their lives evolve to the asymptotic giant branch (AGB) and occupy the region of the Hertzsprung-Russell diagram of high luminosities (typically 5000-50000 $L_{\odot}$ ) and low effective temperatures $(\sim 3000 \mathrm{~K})$. Dust grains can form in the outer layers of the extended atmospheres of AGB stars where the temperatures are low and the densities are enhanced by stellar pulsations. Radiation pressure acting on the grains causes a slow wind to develop $\left(\sim 10 \mathrm{~km} \mathrm{~s}^{-1}\right)$ through which the star looses mass, a process that controls the subsequent evolution of the star (Habing \& Olofsson 2003). The cumulative mass lost during the AGB phase is important for the chemical evolution of galaxies, as the atmospheric composition of AGB stars is modified by dredgeups, which bring elements synthesized in the stellar interior to the surface (e.g. Habing 1996).
Despite a qualitative understanding of the AGB mass-loss process, models are not yet able to predict the mass-loss history of an AGB star from first principles. Both the magnitude of the mass loss and its variation in time are still unanswered questions. For instance, model calculations show that the winds of oxygen-rich AGB stars cannot be driven by absorption of photons. The reason is that iron-bearing silicates that have large near-infrared absorption cross-sections to acquire enough momentum also heat up very efficiently and cannot exist in the wind acceleration region (Woitke 2006). A solution to this problem was proposed by Höfner (2008), who suggested that refractory species with small near-infrared absorption cross-sections - that can exist close to the star - can still transfer momentum to the wind by means of scattering, if the grains can grow to a few tenths of a micron in diameter. Indeed, in a more comprehensive study of the possible wind-drivers in oxygen-rich 
AGB stars, Bladh et al. (2013) found that the dust species expected to form in an oxygen-rich environment are not able to drive the wind via the absorption of photons. The nature of the grains responsible for driving the wind and the particulars of the wind-driving mechanism are still widely discussed (e.g. Norris et al. 2012; Bladh \& Höfner 2012; Bladh et al. 2013; Gail et al. 2013; Karovicova et al. 2013). From wind-driving calculations, the preferred wind-driving candidates are silicate grains (Höfner 2008; Bladh \& Höfner 2012). However, amorphous aluminum oxide is also found to be an important component in general oxygen-rich AGB stars with low mass-loss rates (e.g. Lorenz-Martins \& Pompeia 2000; Speck et al. 2000; Heras \& Hony 2005; Karovicova et al. 2013). The latter might act as a seed for further grain growth and be crucial for starting the wind, despite their low abundance. Aluminum oxide has been suggested to form in the extended atmospheres of oxygen-rich late-type stars both on the basis of grain condensation calculations (e.g. Woitke 2006) and observations (e.g. Verhoelst et al. 2006; Zhao-Geisler et al. 2011). MIDI visibilities of RT Vir, for instance, point to the presence of amorphous $\mathrm{Al}_{2} \mathrm{O}_{3}$ close to the star (at $\sim 1.5 R_{\star}$; Sacuto et al. 2013). However, dust excess spectral fits often require a too large amount of this grain species to match the observed infrared emission; so large that it often implies a super-solar aluminum abundance in the outflow (e.g. Karovicova et al. 2013).

We model the dust envelope of W Hya, a close-by oxygenrich AGB star with a fairly low mass-loss rate (Khouri et al. 2014a, henceforth Paper I) for which a wealth of observational data is available. These data probe the wind structure of W Hya on spatial scales ranging from tens of mas to minutes of arc and reveal grains with radii of about $0.3 \mu \mathrm{m}$ at 40 mas (or $2 R_{\star}$ ) seen in scattered light (Norris et al. 2012); an inner radius for the amorphous silicate emission of 500 mas (or $25 R_{\star}$; Zhao-Geisler et al. 2011), and a mass loss that is variable on dynamical timescales of thousands of years (Hawkins 1990; Cox et al. 2012) that resembles the formation of detached-shells (exclusively) seen around carbon-rich AGB stars (e.g. Olofsson et al. 1990, 1996). Although W Hya has previously been studied extensively, the models obtained for the dust excess only focused on the emission from the inner wind (e.g. Heras \& Hony 2005; Justtanont et al. 2004; Khouri et al. 2014a). None have accounted for the scattered light fractions seen by Norris et al. or the inner emission radius of the amorphous silicates reported by Zhao-Geisler et al., however. Furthermore, all dust emission models obtained so far require super-solar aluminum abundances in the dust when compared to the gas-phase mass-loss rate.

Based on the strong evidence for amorphous $\mathrm{Al}_{2} \mathrm{O}_{3}$ emission coming from very close to the star and the super-solar abundances in the wind that are supposedly needed to account for the amorphous $\mathrm{Al}_{2} \mathrm{O}_{3}$ emission, we consider in our model a gravitationally bound shell of amorphous $\mathrm{Al}_{2} \mathrm{O}_{3}$ grains to be the main contributor to the emission at wavelengths around $12 \mu \mathrm{m}$. We combine this gravitationally bound shell with an outflow to obtain a dust model that fits the wealth of information on the dusty wind of WHya. The properties and spatial distribution of the dust in this model are discussed in the context of wind driving, as well as the time variability of the mass-loss on timescales of millennia.

In Sect. 2 we detail our modelling strategy and the observations of the dust envelope of W Hya. The modelling procedure and fits are described in Sect. 3. We discuss our results in Sect. 4 and provide a summary in Sect. 5 .

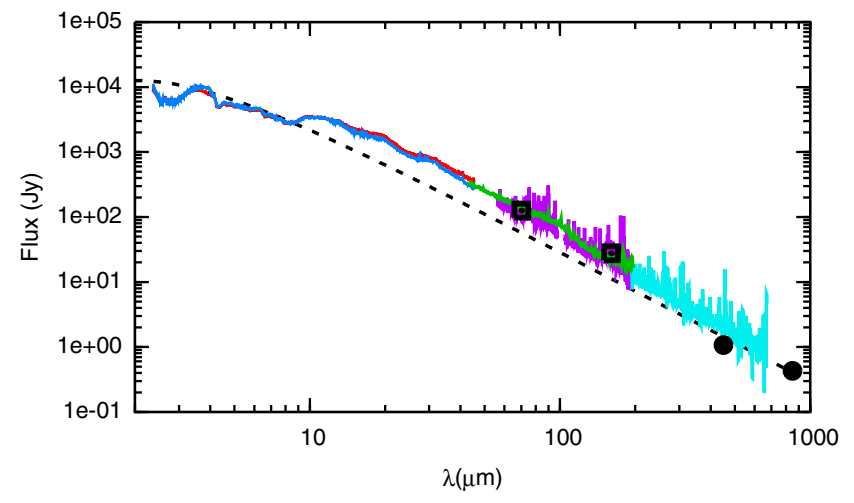

Fig. 1. Compilation of all thermal emission observations of the dust in W Hya. The red and blue solid lines are the ISO SWS spectra from 1996 and 1997, respectively, the green solid line is the averaged ISO LWS spectrum, the purple and light blue solid lines are the PACS and SPIRE spectra, and the black squares and black circles depict the PACS and SCUBA photometric measurements. A black-body spectrum of $2500 \mathrm{~K}$ and $5400 L_{\odot}$ is overplotted to guide the eye (black dashed line).

\section{Observations of the dust envelope of W Hya}

In the following subsections, we present the dataset used in our study to constrain the present-day mass-loss rate. The observations used to model the recent dust mass-loss history are presented in Sect. 3.7.

\subsection{Observed infrared spectra}

W Hya was observed by the Short-Wavelength Spectrometer (SWS, de Graauw et al. 1996) and the Long-Wavelength Spectrometer (LWS, Clegg et al. 1996) onboard the Infrared Space Observatory (ISO, Kessler et al. 1996) and by the Photodetector Array Camera and Spectrometer (PACS, Poglitsch et al. 2010) and the Spectral and Photometric Imaging Receiver (SPIRE, Griffin et al. 2010) onboard Herschel Space Observatory (Pilbratt et al. 2010). A spectrum covering the full range of the instruments was obtained with both PACS and SPIRE. The PACS observations were carried out in different epochs for the blue and red band. The full spectral range of SPIRE was covered in one observation run (see also Table 1). Two spectra were measured with SWS and three with LWS. The two SWS spectra were taken almost one pulsation period apart (Table 1). We obtained the reduced 1997 spectrum from Justtanont et al. (2004) and the 1996 spectrum from the Sloan et al. (2003) database. Two LWS observations were also taken roughly one period apart, with a third taken near maximum visual light. We obtained the highly processed data products of the three spectra from the ISO archive, in which the problem of near-infrared leakage is corrected. The spectrum obtained in February 1997 has an unreliable baseline, especially at long wavelengths, and was not included in our analysis. The other two LWS spectra show very similar flux levels (displaying differences of about $10 \%$ ) and shapes. We have averaged these two spectra before fitting our models. All the observed spectra are shown in Fig. 1; the two SWS spectra differ slightly in flux level, revealing differences of up to $20 \%$, but are very similar in shape. The PACS and SPIRE flux levels agree well with those of LWS when the different fields of view and the PACS far-infrared maps are taken into account (see Sect. 3).

The dust components seen in the ISO spectrum of W Hya are better visualized if we subtract the expected stellar continuum 
T. Khouri et al.: Dusty wind of W Hydrae

Table 1. Overview of the observations of the dust shell of W Hya using different telescopes.

\begin{tabular}{|c|c|c|c|c|c|c|}
\hline Instrument & Date & $\Phi$ & Ref. & $\lambda[\mu \mathrm{m}]$ & FOV [“×”] & Identifier \\
\hline ISO SWS 1 & 14-02-1996 & 0.12 & Sloan et al. (2003) & $2.36-45.39$ & $14 \times 20$ to $17 \times 40$ & 08902004 \\
\hline ISO SWS 2 & 07-01-1997 & 0.17 & Justtanont et al. (2004) & $2.36-45.39$ & $14 \times 20$ to $17 \times 40$ & 41800303 \\
\hline ISO LWS 1 & 07-02-1996 & 0.1 & 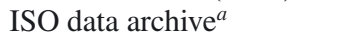 & $43.05-195.5$ & $80 \times 80$ to $70 \times 70$ & 08200208 \\
\hline ISO LWS 2 & 05-02-1997 & 0.1 & ISO data archive ${ }^{a}$ & $43.05-195.5$ & $80 \times 80$ to $70 \times 70$ & 44700672 \\
\hline ISO LWS 3 & 02-08-1997 & 0.6 & ISO data $_{\text {archive }}{ }^{a}$ & $43.05-195.5$ & $80 \times 80$ to $70 \times 70$ & 62500572 \\
\hline PACS Spec B2A & 09-07-2011 & 0.8 & Khouri et al. (2014a) & $55-73$ & $30 \times 30$ & 1342212604 \\
\hline PACS Spec R1A & 09-07-2011 & 0.8 & Khouri et al. (2014a) & $102-146$ & $30 \times 30$ & 1342212604 \\
\hline PACS Spec B2B & 14-01-2011 & 0.4 & Khouri et al. (2014a) & $70-105$ & $30 \times 30$ & 1342223808 \\
\hline PACS Spec R1B & 14-01-2011 & 0.4 & Khouri et al. (2014a) & $140-210$ & $30 \times 30$ & 1342223808 \\
\hline SPIRE Spec & 09-01-2010 & 0.27 & Khouri et al. (2014a) & $124-671$ & $37 \times 37$ to $18 \times 18$ & 1342189116 \\
\hline PACS Image 70 & 08-02-2011 & 0.47 & Cox et al. (2012) & $60-85$ & $1440 \times 1440$ & 1342213848 \\
\hline PACS Image 160 & 08-02-2011 & 0.47 & Cox et al. (2012) & $130-210$ & $1440 \times 1440$ & 1342213849 \\
\hline SCUBA & & & & 450 and 850 & & \\
\hline VLT - MIDI & 04-2007 - 09-2009 & - & Zhao-Geisler et al. (2011) & $8-13$ & & \\
\hline VLT - NACO & 03-2009 and 06-2010 & $0.2^{b}$ & Norris et al. (2012) & $1.04,1.24$ and 2.06 & & \\
\hline
\end{tabular}

Notes. The phase $\Phi$ of the pulsation period is given based on observations of visible light made available by the AAVSO (American Association of Variable Star Observers) at http://www. aavso.org/; ${ }^{(a)}$ http://iso.esac.esa.int/iso/ida/; ${ }^{(b)}$ Visual phase given by Norris et al. (2012).

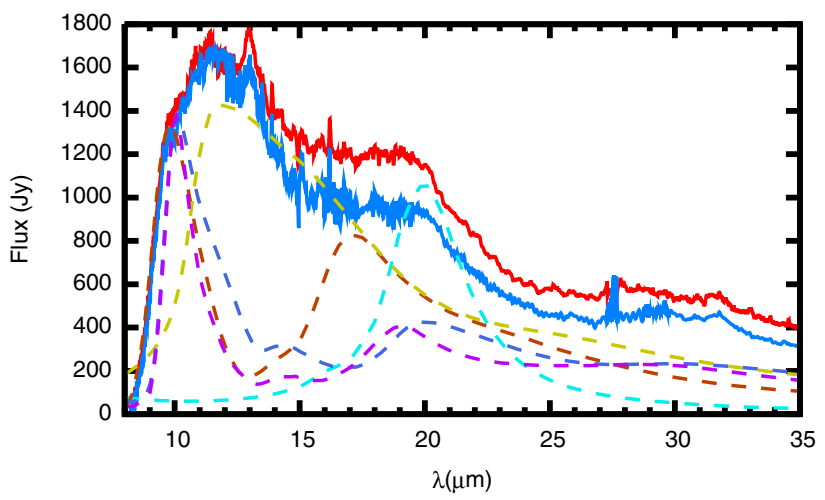

Fig. 2. Continuum-subtracted ISO SWS spectra from 1996 and 1997 are shown by the red and blue lines. The opacity curves of amorphous $\mathrm{Al}_{2} \mathrm{O}_{3}, \mathrm{MgFeSiO}_{4}, \mathrm{FeO}, \mathrm{Ca}_{2} \mathrm{Mg}_{0.5} \mathrm{Al}_{2} \mathrm{Si}_{1.5} \mathrm{O}_{7}$ and $\mathrm{Ca}_{2} \mathrm{Al}_{2} \mathrm{SiO}_{7}$ are shown by the yellow, light brown, light blue, purple, and blue dashed lines, respectively. The opacity curves are scaled to compared them with the stellar-continuum-subtracted spectrum.

from the observed spectrum. We used the stellar parameters and distance from Paper I, $T_{\text {eff }}=2500 \mathrm{~K}, L=5400 L_{\odot}$ and $78 \mathrm{pc}$, to estimate the stellar continuum. In Fig. 2, we compare the opacity curves of the dust species considered by Cami (2002) and Heras \& Hony (2005) and that of $\mathrm{Ca}_{2} \mathrm{Mg}_{0.5} \mathrm{Al}_{2} \mathrm{Si}_{1.5} \mathrm{O}_{7}$ to the two stellar-continuum-subtracted SWS spectra. A combination of amorphous silicates, amorphous $\mathrm{Al}_{2} \mathrm{O}_{3}$, and $\mathrm{Mg}_{0.1} \mathrm{Fe}_{0.9} \mathrm{O}$ can qualitatively account for the emission seen between $9 \mu \mathrm{m}$ and $25 \mu \mathrm{m}$. From $\sim 27 \mu \mathrm{m}$ to $\sim 32 \mu \mathrm{m}$, however, a feature is observed that cannot be reproduced by these dust species. We refer to this feature as the broad $30 \mu \mathrm{m}$ feature. Fabian et al. (2001) identified a narrow feature at $32 \mu \mathrm{m}$ that they assigned to spinel, but this is different from the broad $30 \mu \mathrm{m}$ feature we refer to here.

\subsection{Interferometric observations}

The envelope of W Hya was studied with the Masked AperturePlane Interference Telescope (MAPPIT) at the $3.9 \mathrm{~m}$ AngloAustralian Telescope over the wavelength range $0.65-1.0 \mu \mathrm{m}$ by Ireland et al. (2004). The authors found an increase in measured angular size from $\sim 20$ mas at $1 \mu \mathrm{m}$ to $\sim 35$ mas at shorter wavelengths, which they proposed to be due the scattering of light by dust grains. Later observations of W Hya by Norris et al. (2012) using aperture-masked polarimetric interferometry with the NACO instrument on the VLT confirmed the existence of a shell of scattering dust particles around the star with a radius of about 37.5 mas, or $\sim 2 R_{\star}$. The authors considered a population of large grains with uniform radii in their model, as their technique does not allow them to constrain the presence of small grains, and found the radius of the scattering particles to be $0.316 \pm 0.004 \mu \mathrm{m}$. Although the composition of the dust could not be determined, the authors suggested that they may be composed of iron-free silicates such as forsterite $\left(\mathrm{Mg}_{2} \mathrm{SiO}_{4}\right)$ or enstatite $\left(\mathrm{MgSiO}_{3}\right)$, while corundum $\left(\mathrm{Al}_{2} \mathrm{O}_{3}\right)$ is a possible candidate as well.

Zhao-Geisler et al. (2011) monitored W Hya using MIDI at the VLT. The authors found that the silicate emission seen at $10 \mu \mathrm{m}$ in the ISO spectrum must come from a region with an inner radius larger than 500 mas, corresponding to 25 times the stellar radius or 12 times the scattering shell radius as estimated by Norris et al. (2012). This does not exclude the possibility that silicates condense close to the star, as the iron-free silicates proposed by Norris et al. (2012) to be the scattering agent are transparent in the near infrared and can remain cold $(\sim 700 \mathrm{~K})$ and invisible even at two stellar radii. Such grains would not necessarily produce a signature in the thermal infrared (see Sect. 3.4).

\subsection{Gas-phase wind model}

In Paper I, the present-day gas mass-loss rate of W Hya was determined to be $(1.3 \pm 0.6) \times 10^{-7} M_{\odot} \mathrm{yr}^{-1}$ by fitting the strengths and shapes of the $\mathrm{CO}$ ground-vibrational state, pure-rotational transitions observed by Herschel. This mass-loss rate places constraints on the amount of dust that can form in the wind assuming a solar composition. More stringent limits on the amount of silicates that may form around W Hya were set by Khouri et al. (2014b), who modelled the $\mathrm{SiO}$ gas-phase emission lines observed by Herschel using the wind model obtained in Paper I. The authors found that about $60 \%$ of the silicon in the outflow of W Hya remains in the gas phase. 


\section{Towards a comprehensive present-day mass-loss model}

Our modelling strategy consists of dealing with the different spatial and compositional components of the wind in an independent way first. This approach can be followed as the wind of W Hya is optically thin at the wavelengths where dust emission is important. We start by modelling the innermost observed dust component, namely the scattered light fractions and the amorphous $\mathrm{Al}_{2} \mathrm{O}_{3}$ emission. Then, we model the silicate emission envelope, whose inner radius is at ten times the distance from where the scattered light is seen (Zhao-Geisler et al. 2011). Finally, we compare our best inner wind model with the PACS $70 \mu \mathrm{m}$ image, which provides constraints on both the dust distribution close to the star and at scales up to a few arcminutes (Cox et al. 2012). In our modelling efforts we aim to reproduce the following observations:

- The scattered light fractions as measured by Norris et al. (2012).

- The ISO dust spectrum.

- The lower limit of 500 mas on the inner radius of the silicate emission shell set by Zhao-Geisler et al. (2011).

- The limits set by the gas-phase model of Paper I and Khouri et al. (2014b) on the amount of elements available for dust formation in the wind.

- The broadness of the $70 \mu \mathrm{m}$ radial brightness distribution seen by PACS (Cox et al. 2012).

\subsection{MCMax and model assumptions}

To solve the continuum radiative transfer in the wind of W Hya, we used the code MCMAX (Min et al. 2009). The code calculates the dust spectrum, images of the envelope at different wavelengths, and the fraction of light scattered by the dust particles, all of which are used to constrain our model. The dust envelope was assumed to be spherically symmetric. To derive dust opacities from optical constants, we applied a distribution of hollow spheres (DHS, Min et al. 2003) approximation to represent the particles shapes. We used the direction-dependent scattering phase-function in our calculations, since particles with radii in the range considered scatter light non-isotropically. This was done by considering the full angle-dependent Mueller matrix for each scattering event. The temperature of the dust grains was set by assuming radiative equilibrium. For multiple dust species it may be computed assuming the different types of grains to be either in isolation or in thermal contact. In the case of thermal contact, the absorbed energy is distributed over the constituents of the grain and a single grain temperature is computed such that the emitted energy matches the absorbed energy. We specify throughout the text whenever thermal contact is considered.

To model the amorphous $\mathrm{Al}_{2} \mathrm{O}_{3}$ emission, we used three sets of optical constants, those for porous and compact particles given by Begemann et al. (1997) and those of aerosil particles measured by Koike et al. (1995). The latter measurements were carried out for wavelengths between 0.5 and $500 \mu \mathrm{m}$. The optical constants obtained by Begemann et al. (1997) were measured between 7.7 and $500 \mu \mathrm{m}$ and lack data at near-infrared and optical wavelengths. As these short wavelength data are important for determining the temperature of the grains, we used the optical constants given by Koike et al. (1995) at $\lambda<7.7 \mu \mathrm{m}$. To model the silicate emission, we retrieved data for silicate dust species from the JENA database of optical constants from the works of Jäger et al. (1994), Dorschner et al. (1995), Mutschke et al. (1998), and Jäger et al. (2003). The dust species that are considered are given in Table A.1. For many of the silicate species the optical constants are available only down to about $6 \mu \mathrm{m}$. As most of the radiation that heats the grains is absorbed at shorter wavelengths, we considered the near-infrared optical constants of these dust species to be equal to that of $\mathrm{Mg}_{0.4} \mathrm{Fe}_{0.6} \mathrm{SiO}_{3}, \mathrm{Mg}_{0.8} \mathrm{Fe}_{0.2} \mathrm{SiO}_{3}$ or $\mathrm{MgSiO}_{3}$, selecting out of these three the species that best matches the iron content.

We considered W Hya to be at 78 parsecs, to have a luminosity of $5400 L_{\odot}$ and the stellar spectrum to be that of a black-body with $2500 \mathrm{~K}$, following our assumptions in Paper I.

\subsection{Previous dust model}

The model obtained in Paper I for the wind of W Hya (shown in Fig. 10) adopted the dust components identified by Justtanont et al. (2004). In Paper I, the region of the ISO spectrum between 8 and $30 \mu \mathrm{m}$ of the ISO spectrum was fitted, but no attempt was made to fit the $13 \mu \mathrm{m}$ feature as its carrier is still uncertain (see Posch et al. 1999; Zeidler et al. 2013, and references therein). A total dust mass-loss rate of $2.8 \times 10^{-10} M_{\odot}$ year $^{-1}$ was obtained. The dust composition by mass is $58 \%$ astronomical silicates (Justtanont \& Tielens 1992), 34\% amorphous aluminum oxide $\left(\mathrm{Al}_{2} \mathrm{O}_{3}\right)$, and $8 \%$ magnesium-iron oxide $(\mathrm{MgFeO})$. Optical constants for the latter species were retrieved from the database of the University of Jena from the work of Henning et al. (1995). For amorphous aluminum oxide, the authors have used data for porous particles from Begemann et al. (1997).

\subsection{Amorphous $\mathrm{Al}_{2} \mathrm{O}_{3}$ emission}

As pointed out in the introduction, the amorphous $\mathrm{Al}_{2} \mathrm{O}_{3}$ mass fraction of the total dust needed to explain the mid-infrared excess of low mass-loss rate AGB stars is often very high (e.g. Lorenz-Martins \& Pompeia 2000; Speck et al. 2000; Heras \& Hony 2005; Karovicova et al. 2013). The maximum amount of a given dust species that can exist in the stellar wind is determined by the availability of its least abundant element. In the case of amorphous $\mathrm{Al}_{2} \mathrm{O}_{3}$ this limit is set by aluminum, whose mass abundance relative to hydrogen is $7.6 \times 10^{-5}$ for a solar composition (Asplund et al. 2009). If all aluminum atoms in the wind of W Hya were used to form amorphous $\mathrm{Al}_{2} \mathrm{O}_{3}$ grains, the mass-loss rate of this dust species alone would be $\dot{\mathrm{M}}_{\mathrm{Al}_{2} \mathrm{O}_{3}}=1.3 \times 10^{-7} \times 7.6 \times 10^{-5} / 0.53=1.9 \times 10^{-11} M_{\odot}$ year $^{-1}$, where 0.53 is the fraction of the mass of amorphous $\mathrm{Al}_{2} \mathrm{O}_{3}$ in aluminum atoms and $1.3 \times 10^{-7} M_{\odot}$ year $^{-1}$ the gas mass-loss rate. Assuming the specific weight of amorphous $\mathrm{Al}_{2} \mathrm{O}_{3}$ to be $4.0 \mathrm{~g} \mathrm{~cm}^{-3}$, we computed models with this amorphous $\mathrm{Al}_{2} \mathrm{O}_{3}$ mass loss, condensing at two stellar radii. At $12 \mu \mathrm{m}$, the excess emission produced by these models is only $5 \%$ of what is seen in the ISO spectrum (see Fig. 3). These calculations depend on the adopted grain model, the optical constants used, and the assumed location of the onset of the wind. We investigated these parameters using the wind model derived in Paper I by modifying the opacities for amorphous $\mathrm{Al}_{2} \mathrm{O}_{3}$ at short wavelengths and varying the inner radius of the dust envelope accordingly. We find that even an increase in the near-IR opacity of amorphous $\mathrm{Al}_{2} \mathrm{O}_{3}$ by a factor of 10 increases the emission at $12 \mu \mathrm{m}$ by only about a factor of four. Increasing the near-IR opacity also leads to an important contribution of amorphous $\mathrm{Al}_{2} \mathrm{O}_{3}$ emission at wavelengths shorter than $10 \mu \mathrm{m}$. We conclude that the emission attributed to amorphous $\mathrm{Al}_{2} \mathrm{O}_{3}$ in W Hya cannot be accounted for by such grains in the present-day wind alone. 


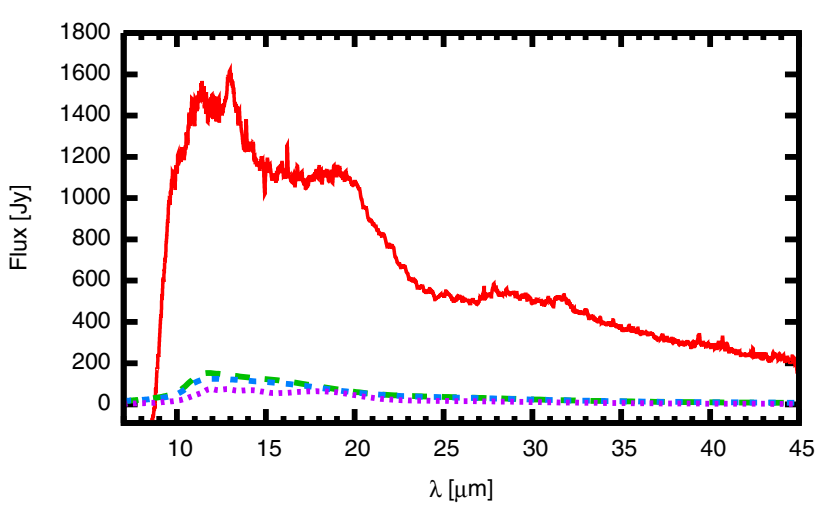

Fig. 3. Models considering full condensation of aluminum in a solarcomposition outflow compared to the stellar-continuum-subtracted infrared excess (solid red). The aerosil $\mathrm{Al}_{2} \mathrm{O}_{3}$ particles, porous $\mathrm{Al}_{2} \mathrm{O}_{3}$ particles and, compact $\mathrm{Al}_{2} \mathrm{O}_{3}$ particles are represented by the dotted purple, short dashed blue and long dashed green lines. None of the considered species produces strong enough emission at $12 \mu \mathrm{m}$.

\subsection{Gravitationally bound dust shell}

We calculated models for a gravitationally bound dust shell (GBDS) located at about $2.0 R_{\star}$ to account for the amorphous $\mathrm{Al}_{2} \mathrm{O}_{3}$ emission and the scattered light fractions. The GBDS is composed of grains located in the region between the radius of onset of condensation of amorphous $\mathrm{Al}_{2} \mathrm{O}_{3}$ and that at which the radiation pressure on the dust grains overcomes the pull of gravity. Since we do not calculate the dust condensation nor the transfer of momentum to the dust grains, the inner and outer radii of this region are free parameters in our models. In the GBDS the grains must reach the optimal size for scattering, at which radiation forces can accelerate them. We therefore expect to find a distribution of sizes ranging from small grains, with radii of the order of $0.01 \mu \mathrm{m}$ to larger grains with radii of about $0.3 \mu \mathrm{m}$. As an instructive exercise, we can approximate the size distribution by a mix of two particle sizes, small and large grains. These can be studied somewhat independently based on the observations available: large grains dominate the scattering of radiation, and small grains do not contribute to the scattered light and probably dominate the infrared emission. We have considered small grains of $0.03 \mu \mathrm{m}$ and large grains of $0.3 \mu \mathrm{m}$.

We considered the grains in the GBDS to be composed of either amorphous $\mathrm{Al}_{2} \mathrm{O}_{3}$ or $\mathrm{Mg}_{2} \mathrm{SiO}_{4}$. The amorphous $\mathrm{Al}_{2} \mathrm{O}_{3}$ emission can be fitted equally well with large and small grains of this dust species. Silicate emission from close to the star is not detected (Zhao-Geisler et al. 2011) but this does not necessarily mean that silicates are absent. Iron-free silicates (such as $\mathrm{Mg}_{2} \mathrm{SiO}_{4}$ ) can exist at close distances from the surface because they are translucent in the optical and near-infrared and therefore remain relatively cold. This does imply that we will not be able to constrain the mass in small $\mathrm{Mg}_{2} \mathrm{SiO}_{4}$ grains with our models. A fit to the observed scattered light fractions requires large grains and in principle can be obtained with either type of grain. Such a fit sets an upper limit on the amount of large grains of the given species.

We considered the amorphous $\mathrm{Al}_{2} \mathrm{O}_{3}$ emission to originate from a stationary shell extending from 1.7 to $2.0 R_{\star}$. The inner radius was set at that at which the small amorphous $\mathrm{Al}_{2} \mathrm{O}_{3}$ grains reach temperatures of $1400 \mathrm{~K}$. The outer radius was assumed to be that found by Norris et al. (2012) for the origin of the scattered light. In principle the grains in this region may be created and destroyed as material rises and falls as a consequence of stellar pulsations, but that does not affect our results. Our modelling shows that the amount of emission is only mildly sensitive to the exact value of the boundaries or to a radial density gradient within the shell, but mostly to the total mass of emitting amorphous $\mathrm{Al}_{2} \mathrm{O}_{3}$. The scattered light fractions were fitted considering a shell with an inner radius of $2.0 R_{\star}$ (Norris et al. 2012) and thickness of $0.1 R_{\star}$, that is, enclosing the shell from where amorphous $\mathrm{Al}_{2} \mathrm{O}_{3}$ originates. In the context of our model, this shell of large grains may be understood as being the interface between the GBDS and the outflow.

A population of only small amorphous $\mathrm{Al}_{2} \mathrm{O}_{3}$ grains reaches temperatures of $1250 \mathrm{~K}$ at $2.0 R_{\star}$, while large amorphous $\mathrm{Al}_{2} \mathrm{O}_{3}$ grains reach $1500 \mathrm{~K}$ at the same distance. The higher temperature of large amorphous $\mathrm{Al}_{2} \mathrm{O}_{3}$ grains has two causes. First, the mass-absorption coefficient between 0.2 and $2 \mu \mathrm{m}$ is higher for the large grains than for small grains. Consequently, large grains absorb more radiation by mass than their smaller counterparts and reach higher temperatures. Second, the scattering of photons by large grains contributes to the diffuse radiation field and the density of short-wavelengths photons $(\lambda \lesssim 2 \mu \mathrm{m})$ significantly increases. The stronger radiation field at shorter wavelengths leads to more energy absorbed per time per grain and to a higher dust temperature. This second effect also causes small amorphous $\mathrm{Al}_{2} \mathrm{O}_{3}$ particles to have higher temperatures when placed in a region with $0.3 \mu \mathrm{m}$ grains. Assuming solar composition and full aluminum condensation, a shell of small amorphous $\mathrm{Al}_{2} \mathrm{O}_{3}$ grains between 1.7 and $2.0 R_{\star}$ requires a gas mass of $\sim 1.4 \times 10^{-5} M_{\odot}$ to reproduce the amorphous $\mathrm{Al}_{2} \mathrm{O}_{3}$ emission seen in the ISO spectrum. Such a shell produces an infrared spectrum indistinguishable from the amorphous $\mathrm{Al}_{2} \mathrm{O}_{3}$ models shown in Fig. 5 for a population with a range in grain radii.

The mass in large grains needed to reproduce the scattering is $4.9 \times 10^{-10} M_{\odot}$ for amorphous $\mathrm{Al}_{2} \mathrm{O}_{3}$ and $3.6 \times 10^{-10} M_{\odot}$ for $\mathrm{Mg}_{2} \mathrm{SiO}_{4}$ grains, assuming specific weights of $4 \mathrm{~g} \mathrm{~cm}^{-3}$ and $3.2 \mathrm{~g} \mathrm{~cm}^{-3}$, respectively. By assuming a solar composition, full aluminum condensation, and 35\% of silicon condensation (as to be consistent with the available silicon for dust condensation derived from the gas-phase analysis; Khouri et al. 2014b), we obtain gas masses of $3.5 \times 10^{-6} M_{\odot}$ and $2.3 \times 10^{-7} M_{\odot}$ for large grains of amorphous $\mathrm{Al}_{2} \mathrm{O}_{3}$ and $\mathrm{Mg}_{2} \mathrm{SiO}_{4}$, respectively.

If the fit to both the ISO infrared excess and the scattered light fractions are combined, the amorphous $\mathrm{Al}_{2} \mathrm{O}_{3}$ emission and the scattered light fractions can be explained by a single population of amorphous $\mathrm{Al}_{2} \mathrm{O}_{3}$ grains located in a shell with inner and outer radii of 1.7 and $2.1 R_{\star}$, respectively, and with a grain size distribution of the type, $n(a) \propto a^{-3.5}$, where $a$ is the grain radius. This expression corresponds to the standard distribution for grain sizes found in the interstellar medium by Mathis et al. (1977, MRN distribution). The lower limit of the distribution was assumed to be $0.01 \mu \mathrm{m}$ and the maximum grain radius to be that derived by Norris et al., i.e. $0.316 \mu \mathrm{m}$. By assuming solar composition and full aluminum condensation, the total gas mass required for this population of amorphous $\mathrm{Al}_{2} \mathrm{O}_{3}$ is $\sim 1.2 \times 10^{-5} M_{\odot}$. The differences in the amorphous $\mathrm{Al}_{2} \mathrm{O}_{3}$ mass needed to reproduce the observed emission only considering small grains or a grain size distribution is due to the different grain temperatures obtained. The fit to the scattered light fractions using an MRN size distribution is very similar to the one obtained with the large-grains-only model, shown in Fig. 4, and is not given. Gradients in the grain size throughout the GBDS may be present and would allow similar quality fits as adopting a size distribution. Infrared spectra for an MRN distribution of particles adopting different optical constants for amorphous $\mathrm{Al}_{2} \mathrm{O}_{3}$ 


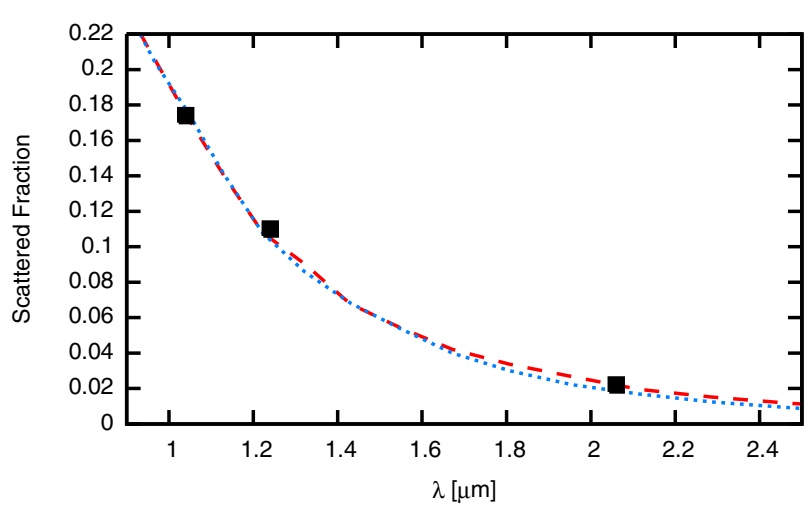

Fig. 4. Scattering fractions for the models with large particles of aerosil $\mathrm{Al}_{2} \mathrm{O}_{3}$ (red long dashed line) and $\mathrm{Mg}_{2} \mathrm{SiO}_{4}$ (blue short dashed line) in the GBDS. The black squares are the observations by Norris et al. (2012).

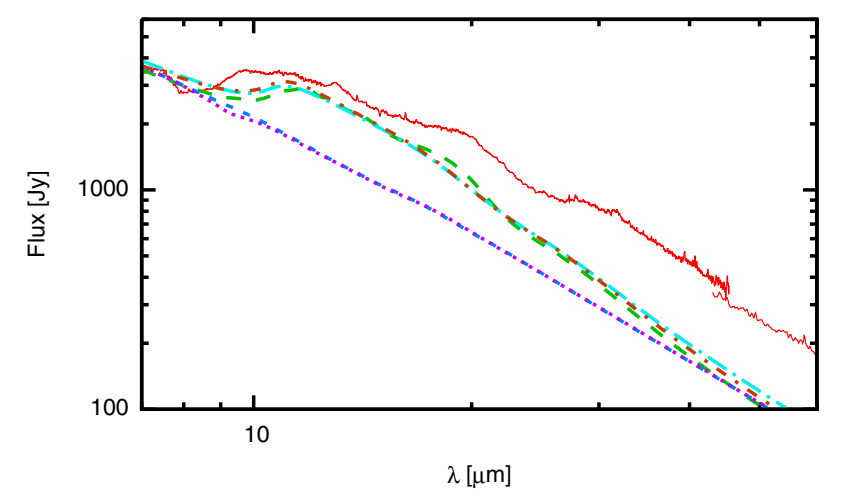

Fig. 5. Spectra of a population of amorphous $\mathrm{Al}_{2} \mathrm{O}_{3}, \mathrm{MgSiO}_{3}$ or $\mathrm{Mg}_{2} \mathrm{SiO}_{4}$ grains compared to the observed ISO spectrum (Sloan et al. 2003). We considered an MRN grain radii distribution for all models shown here. The different lines are for grains consisting of aerosil $\mathrm{Al}_{2} \mathrm{O}_{3}$ particles (green dashed), $\mathrm{Al}_{2} \mathrm{O}_{3}$ porous particles (light blue long dasheddotted), $\mathrm{Al}_{2} \mathrm{O}_{3}$ compact particles (brown short dashed-dotted), $\mathrm{MgSiO}_{3}$ (purple dotted) and $\mathrm{Mg}_{2} \mathrm{SiO}_{4}$ (short dashed blue).

are shown in Fig. 5. The similar amorphous $\mathrm{Al}_{2} \mathrm{O}_{3}$ emission obtained shows that the applied optical constants do not impact our findings significantly either.

If the scattering is due to large $\mathrm{Mg}_{2} \mathrm{SiO}_{4}$ grains, a population of small amorphous $\mathrm{Al}_{2} \mathrm{O}_{3}$ grains is still required to account for the infrared excess emission. When a population of large silicate grains is placed in the shell together with small amorphous $\mathrm{Al}_{2} \mathrm{O}_{3}$ particles, the temperatures are about $700 \mathrm{~K}$ and $1400 \mathrm{~K}$, respectively. When these two species are placed in thermal contact, the resulting temperature will depend on the mass fraction of each species in a given grain. Assuming full aluminum and $35 \%$ silicon condensation implies that $8 \%$ of the mass is in amorphous $\mathrm{Al}_{2} \mathrm{O}_{3}$ and $92 \%$ in $\mathrm{Mg}_{2} \mathrm{SiO}_{4}$. In this case, the equilibrium temperature is about $800 \mathrm{~K}$ and the silicate features would remain undetected, in agreement with the limit set for the inner radius of amorphous silicate emission of $\sim 40$ AU. Therefore, a scenario in which small amorphous $\mathrm{Al}_{2} \mathrm{O}_{3}$ grains are formed close to the star and in which rapid condensation of $\mathrm{Mg}_{2} \mathrm{SiO}_{4}$ onto the amorphous $\mathrm{Al}_{2} \mathrm{O}_{3}$ seeds occurs as well would be consistent with all observations. In this scenario, the condensation of $\mathrm{Mg}_{2} \mathrm{SiO}_{4}$ could be halted by the onset of the wind.

\subsection{Dust properties in the outflow}

The contribution of amorphous $\mathrm{Al}_{2} \mathrm{O}_{3}$ grains in a GBDS to the infrared spectrum is somewhat independent of the size distribution and the exact location of the shell as the grains would be sufficiently warm for the shape of their emission feature to be independent of temperature for the optical constant adopted by us. Although the opacities of crystalline $\mathrm{Al}_{2} \mathrm{O}_{3}$ show significant variations with temperature (Zeidler et al. 2013), to our knowledge, no studies have been performed that probe the effect of temperature variations on the amorphous $\mathrm{Al}_{2} \mathrm{O}_{3}$ features. In the context of our model, only the strength of the emission is sensitive to temperature, and therefore the total grain mass needed to reproduce the infrared excess is a function of grain temperature. If we assume that the dust shell is optically thin, we may subtract the amorphous $\mathrm{Al}_{2} \mathrm{O}_{3}$ contribution from the ISO spectrum and study the residual spectrum (see Fig. 6). The assumed size distribution of amorphous $\mathrm{Al}_{2} \mathrm{O}_{3}$ grains does not affect the overall shape of the residual spectrum. Uncertainties in the residual spectrum arise from the choice of optical constants for amorphous $\mathrm{Al}_{2} \mathrm{O}_{3}$, the adopted grain model, and variability of the star, but these probably have a modest effect.

The residual spectrum clearly shows the silicate peak at $9.7 \mu \mathrm{m}$, the unidentified $13 \mu \mathrm{m}$ feature, a peak at about $20 \mu \mathrm{m}$ that is probably due to $18 \mu \mathrm{m}$ silicate and/or magnesium-iron oxide emission, and a broad feature around $30 \mu \mathrm{m}$. For simplicity and because of the similarity in the different $\mathrm{Al}_{2} \mathrm{O}_{3}$-subtracted residual spectra, we only used the ISO spectrum retrieved from the Sloan et al. (2003) database subtracted by the spectrum of aerosil amorphous $\mathrm{Al}_{2} \mathrm{O}_{3}$ grains (Fig. 6; red solid line).

\subsubsection{Fit to the residual spectrum}

The dust species considered in fitting the silicate emission are given in Table A.1. All the species listed have somewhat similar opacity profiles that are characteristic of silicate glasses. Diagnostic parameters such as the peak strength ratio, the central wavelength, and the full-width at half maximum (FWHM) of the peaks can help constrain the composition (Dorschner et al. 1995; Mutschke et al. 1998) and are listed for DHS particles with $0.3 \mu \mathrm{m}$ radius. The position of the two silicate peaks measured from the $\mathrm{Al}_{2} \mathrm{O}_{3}$-subtracted residual spectra are $9.94 \pm 0.5 \mu \mathrm{m}$ and $19.7 \pm 0.5 \mu \mathrm{m}$. It is important to note that the peak at $19.7 \mu \mathrm{m}$ might be produced by a combination of silicate and $\mathrm{Mg}_{\mathrm{x}} \mathrm{Fe}_{1-\mathrm{x}} \mathrm{O}$ emission, so the measured position should be considered with care.

We recall that the VLT $10 \mu \mathrm{m}$ observations provide a lower limit to the radial distance of the silicate-emitting shell of about 40 AU. Most of the dust species listed in Table A.1 have a low iron content and consequently a low near-infrared opacity. These species would have too low temperatures at such large distances and would not produce detectable infrared flux relative to the bright stellar continuum and emission from the other dust species. As the ratio between the two silicate emission peaks indicates that the grains must be warm $(\sim 500 \mathrm{~K})$, the silicates with low near-infrared opacities, that is, with low iron content, were placed in thermal contact with metallic iron to increase their temperatures. Such metallic iron inclusions are indeed expected to form in circumstellar silicates grains (Gail \& Sedlmayr 1999). We considered models with an iron content per unit mass ranging from 0 to $80 \%$. Accounting for even higher amounts of metallic iron causes the silicate features to become too weak with respect to the continuum emission that the metallic iron produces. Silicate species with significant iron content, such as 


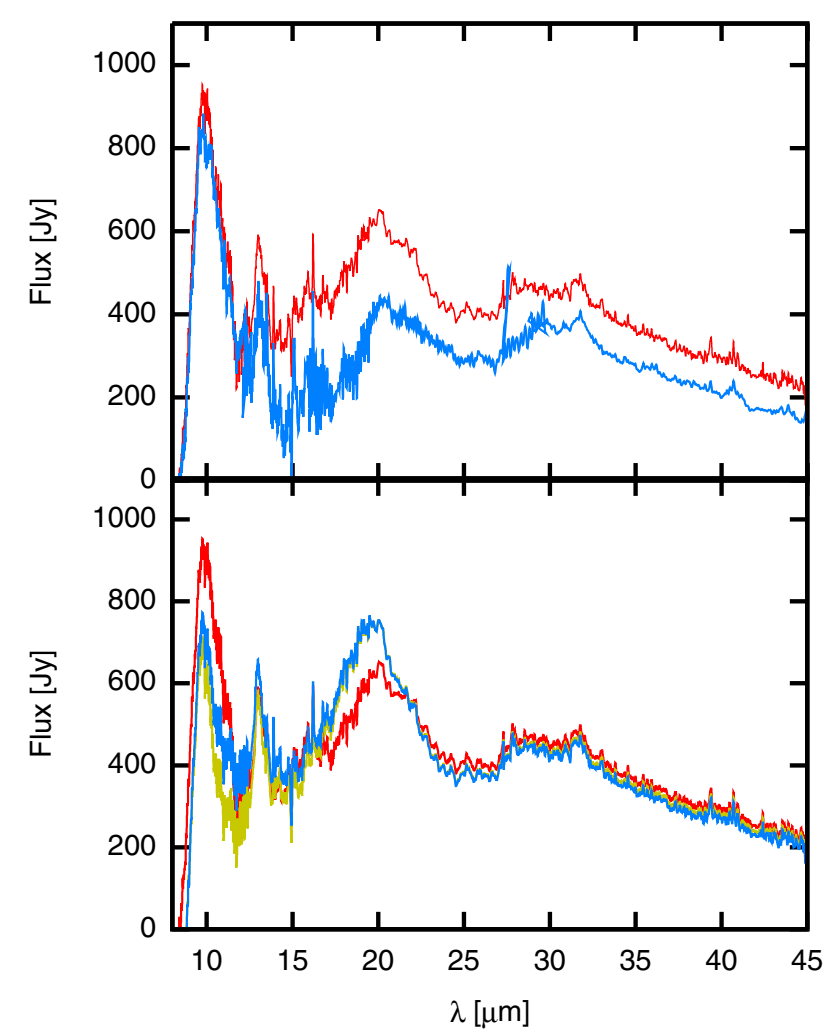

Fig. 6. $\mathrm{Al}_{2} \mathrm{O}_{3}$-subtracted residual infrared spectrum of $\mathrm{W}$ Hya. Upper panel: residuals adopting the optical constants for aerosil particles from Koike et al. (1995) and the ISO spectra from the Sloan et al. (2003) database (in red) and Justtanont et al. (2004; in blue); lower panel: the red curve is the same as in the upper panel. The yellow and blue curves are for residuals assuming compact and porous particles from Begemann et al. (1997).

$\mathrm{MgFeSiO}_{4}$, have high enough temperatures even when placed at $40 \mathrm{AU}$ and require modest or no thermal contact with metallic iron at all.

Models were calculated for silicate envelopes ranging from $40 \mathrm{AU}$ to $1800 \mathrm{AU}$ (i.e. the outer limit given by the field of view of ISO SWS) for all species listed in Table A.1 separately. When in thermal contact with substantial amounts of metallic iron, the silicate grains can be heated up to $400-450 \mathrm{~K}$ at $40 \mathrm{AU}$ and some of the species considered produce peak ratios that reflect the observed ratio. As the temperatures that are reached are still fairly low, species that have relatively high values for the $\kappa_{18 \mu \mathrm{m}}^{\max } / \kappa_{9.7 \mu \mathrm{m}}^{\max }$ are preferred. Furthermore, metallic iron produces emission that fills the trough between the silicate peaks and matches the observed $\mathrm{Al}_{2} \mathrm{O}_{3}$-subtracted residual spectrum. Figure 7 shows the effect of varying the amount of iron in thermal contact with the silicate grains. A higher iron content implies more emission in the silicate trough and blue-wards of the $9.7 \mu \mathrm{m}$ peak. For a given silicate species, the ratio between the red and the blue silicate peaks decreases with an increasing amount of metallic iron inclusions. The dependence is stronger for smaller amounts of iron, between 0 and $50 \%$ per mass.

When using a continuous distribution of ellipsoids (CDE, Bohren \& Huffman 1998; Min et al. 2003) as the grain model for the metallic iron particles, the amount of this species needed to heat the silicates to a given temperature is greatly reduced if compared to models with DHS metallic iron particles. Kemper et al. (2002) also found a large difference between the opacity of

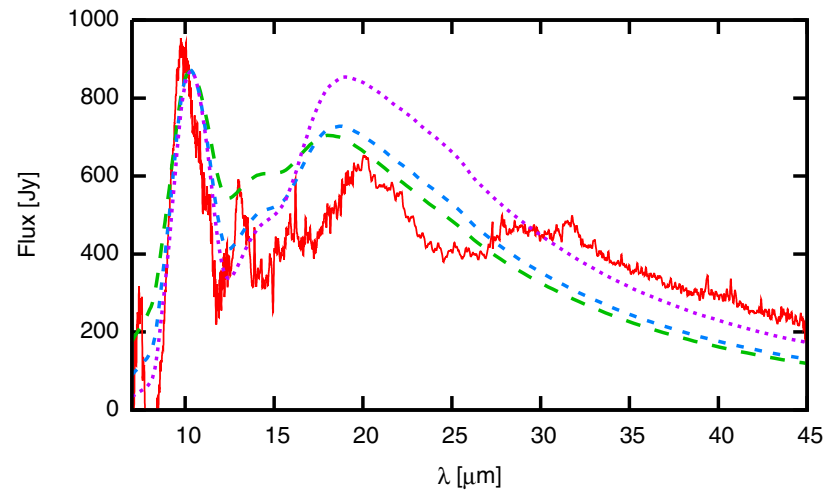

Fig. 7. Models for an envelope composed of $\mathrm{Mg}_{2} \mathrm{AlSi}_{2} \mathrm{O}_{7.5}$ and metallic iron ranging from $40 \mathrm{AU}$ to $1800 \mathrm{AU}$. The different lines are for $30 \%$ (dotted purple), 50\% (short dashed blue) and 70\% (long dashed green) metallic iron content. The $\mathrm{Al}_{2} \mathrm{O}_{3}$-subtracted residual ISO spectrum is shown by the full red line.

CDE metallic iron grains and that obtained using Mie theory. We find the DHS metallic iron model to have an intermediate opacity between these two extremes and discuss the effects of using CDE metallic iron grains in Sect. 4.3.1.

Another feature in the $\mathrm{Al}_{2} \mathrm{O}_{3}$-subtracted residual spectrum worth noting is the broad component at $30 \mu \mathrm{m}$. The mass-loss rate of W Hya varies on larger scales, therefore this emission bump might be thought to be a signature of an abrupt change in mass-loss rate. However, the bump seems too sharp to be produced in such a way. Moreover, this feature is seen in many other ISO spectra of oxygen-rich AGB stars with low massloss rate, suggesting a more fundamental cause. Gervais et al. (1987) studied the infrared properties of silicate glasses and concluded that calcium-bearing silicates have a characteristic band around $30 \mu \mathrm{m}$. From the work of Mutschke et al. (1998), we retrieved the optical data for two Ca-silicates, which do indeed show a feature at $30 \mu \mathrm{m}$. Ca-bearing silicates are expected to form from gas-solid chemical reactions between $\mathrm{Al}_{2} \mathrm{O}_{3}$ grains and calcium and silicon in the gas (Grossman \& Larimer 1974). Another candidate for explaining the feature is pure calciumoxide grains. However, this species produces a rather localized peak, whereas calcium-bearing silicates also provide continuum opacity beyond $30 \mu \mathrm{m}$, which better matches what is seen in the spectrum of W Hya.

As shown in Fig. 8, the calcium-aluminum silicate fits the $\mathrm{Al}_{2} \mathrm{O}_{3}$-subtracted residual spectral shape nicely when in thermal contact with metallic iron. Unfortunately, we were unable to find other optical constants of calcium-bearing silicates in the literature, which potentially could provide a better fit to the $9.7 \mu \mathrm{m}$ silicate peak. As calcium is indeed expected to condense just after $\mathrm{Al}_{2} \mathrm{O}_{3}$, the existence of this dust species in the wind of W Hya can be expected. The model shown in Fig. 8 has a dust mass-loss rate of $3 \times 10^{-10} M_{\odot}$ year $^{-1}$, consisting of $60 \%$ $\mathrm{Ca}_{2} \mathrm{Mg}_{0.5} \mathrm{Al}_{2} \mathrm{Si}_{1.5} \mathrm{O}_{7}$ and $40 \%$ metallic iron, per mass. Further constraints on these quantities are obtained in Sect. 3.6 by fitting the broadness of the PACS $70 \mu \mathrm{m}$ brightness profile.

Even though the fit using $\mathrm{Ca}_{2} \mathrm{Mg}_{0.5} \mathrm{Al}_{2} \mathrm{Si}_{1.5} \mathrm{O}_{7}$ is offset in the $9.7 \mu \mathrm{m}$ region, this single dust species is able to qualitatively reproduce the $\mathrm{Al}_{2} \mathrm{O}_{3}$-subtracted residual spectrum very well. Therefore we conclude that $\mathrm{Ca}_{2} \mathrm{Mg}_{0.5} \mathrm{Al}_{2} \mathrm{Si}_{1.5} \mathrm{O}_{7}$ is the silicate from our set of optical constants that best represents the composition of the silicate dust in the envelope of W Hya. A better fit to the individual features might require a more complex dust envelope structure, which could be linked to the condensation of 


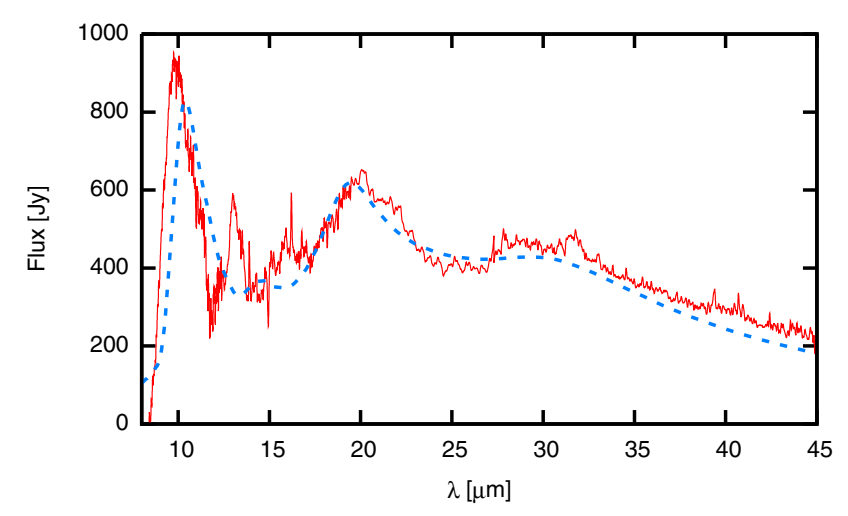

Fig. 8. Fit (dashed blue) to the amorphous $\mathrm{Al}_{2} \mathrm{O}_{3}$-shell-subtracted thermal spectrum (in solid red), adopting $40 \mathrm{AU}$ as the inner boundary of the silicate emitting envelope and a mixture of $40 \%$ metallic iron and $60 \% \mathrm{Ca}_{2} \mathrm{Mg}_{0.5} \mathrm{Al}_{2} \mathrm{Si}_{1.5} \mathrm{O}_{7}$ particles that are in thermal contact.

different elements at different distances from the star, but such a more complex dust model is beyond the scope of this paper. Having this amount of $\mathrm{Ca}_{2} \mathrm{Mg}_{0.5} \mathrm{Al}_{2} \mathrm{Si}_{1.5} \mathrm{O}_{7}$ condense from the outflowing material, however, requires a super-solar abundance of calcium and aluminum. We discuss this in Sect. 4.3.2.

\subsection{Fitting the peak value and broadness of the PACS $70 \mu \mathrm{m}$ image}

When the model consisting of an amorphous $\mathrm{Al}_{2} \mathrm{O}_{3}$ GBDS and $\mathrm{Ca}_{2} \mathrm{Mg}_{0.5} \mathrm{Al}_{2} \mathrm{Si}_{1.5} \mathrm{O}_{7}$ grains in the wind that are in thermal contact with metallic iron (see Fig. 9) is compared to the PACS $70 \mu \mathrm{m}$ radial brightness profile (Cox et al. 2012), the peak value of this profile is not reproduced. The model peak flux is $30 \%$ lower than the observed value and essentially constrains the present-day dust mass-loss. The broadness of the intensity profile in the first few arcseconds constrains the distribution of the bulk of the $70 \mu \mathrm{m}$ emission.

When matching the present-day dust mass loss to the peak strength, the fit to the ISO spectrum becomes poorer. To recover the fit, we shifted the inner radius of the silicate shell from $40 \mathrm{AU}$ to $50 \mathrm{AU}$ and increased the iron content from $40 \%$ to $50 \%$ of the dust mass. The width of the PACS intensity profiles is matched if we decrease the dust mass-loss rate beyond $500 \mathrm{AU}$ by a factor of two, to $2 \times 10^{-10} M_{\odot}$ year $^{-1}$, preserving the dust composition from the inner part. The best model thus consists of a wind composed of $50 \% \mathrm{Ca}_{2} \mathrm{Mg}_{0.5} \mathrm{Al}_{2} \mathrm{Si}_{1.5} \mathrm{O}_{7}$ and $50 \%$ metallic iron in thermal contact and mass-loss rates of $4 \times 10^{-10} M_{\odot}$ year $^{-1}$ between $50 \mathrm{AU}$ and $500 \mathrm{AU}$ and of $2 \times 10^{-10} M_{\odot}$ year $^{-1}$ from $500 \mathrm{AU}$ to $1800 \mathrm{AU}$. The fit to the ISO SWS spectrum of this best model for the outflow combined with the GBDS of amorphous $\mathrm{Al}_{2} \mathrm{O}_{3}$ is shown in Fig. 10. Comparing our dust model and the $\mathrm{CO}$ model (Paper I) yields a gas-to-dust ratio in the inner wind of W Hya of 325 .

\subsubsection{CO envelope}

The mass-loss rate discontinuity at $500 \mathrm{AU}$ is close enough to the star that it might affect the $\mathrm{CO}$ envelope. The model for the CO rotational emission presented in Paper I does not take this variable mass loss into account and therefore has to be revised. In the CO study a fit to both the high- and low-excitation rotational lines was achieved by decreasing the photodissociation radius of $\mathrm{CO}$ by a factor of 2.5 , placing it at about $500 \mathrm{AU}$ from W Hya.

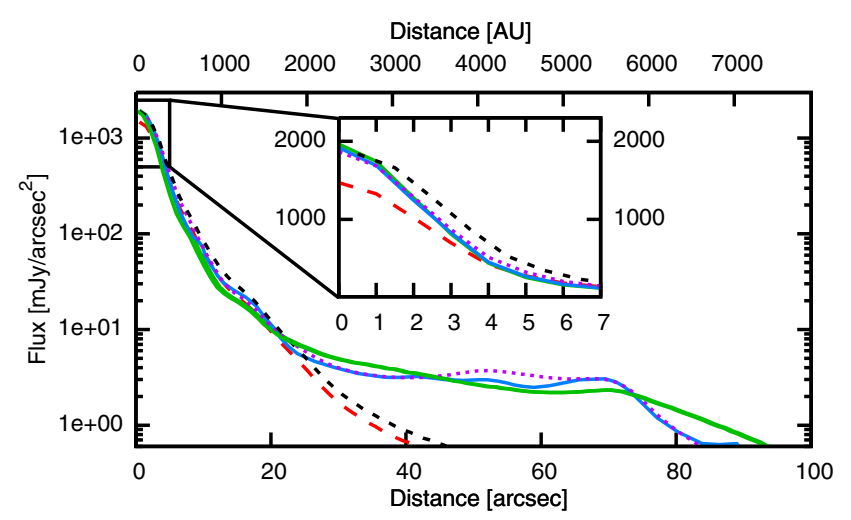

Fig. 9. Fit to the radial intensity profiles observed by PACS at $70 \mu \mathrm{m}$. The blue and green solid lines represent the observed brightness distribution averaged over a narrow range of direction (Cox et al. 2012) and over all directions, respectively. The long dashed red line represents the model with $\dot{M}=3 \times 10^{-10} M_{\odot}$ year $^{-1}$, inner and outer radius of $40 \mathrm{AU}$ and $1800 \mathrm{AU}$, respectively, and with grains composed of $40 \%$ metallic iron and $60 \% \mathrm{Ca}_{2} \mathrm{Mg}_{0.5} \mathrm{Al}_{2} \mathrm{Si}_{1.5} \mathrm{O}_{7}$. The short dashed black line shows the model with $\dot{M}=4 \times 10^{-10} M_{\odot}$ year $^{-1}$, inner and outer radius of $50 \mathrm{AU}$ and $1800 \mathrm{AU}$, respectively, and with grains composed of $50 \%$ metallic iron and $50 \% \mathrm{Ca}_{2} \mathrm{Mg}_{0.5} \mathrm{Al}_{2} \mathrm{Si}_{1.5} \mathrm{O}_{7}$. The dotted purple line represents the model for the recent mass-loss history obtained in Sect. 3.7, with parameters given in Fig. 12 and with grains composed of 50\% metallic iron and $50 \% \mathrm{Ca}_{2} \mathrm{Mg}_{0.5} \mathrm{Al}_{2} \mathrm{Si}_{1.5} \mathrm{O}_{7}$. The radial distance in $\mathrm{AU}$ is given along the upper $x$-axis for an assumed distance of 78 parsecs.

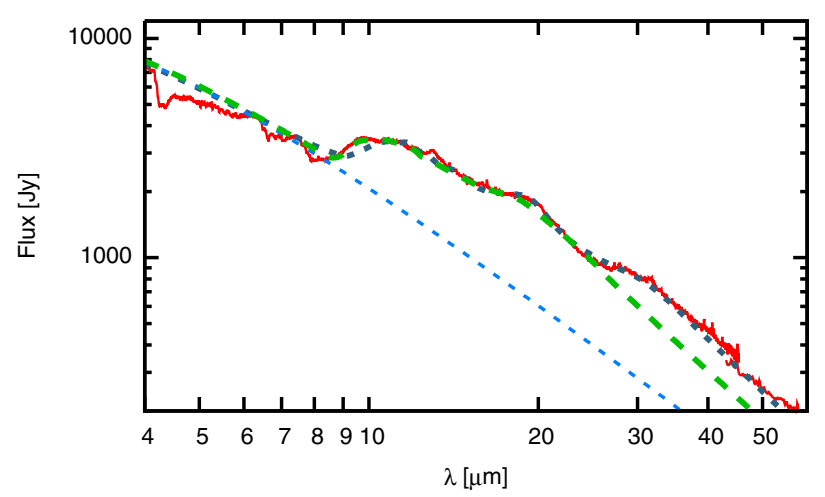

Fig. 10. Overall fit of the best model (short dashed dark blue line) to the ISO SWS spectrum (solid red line). The model consists of the GBDS composed of amorphous $\mathrm{Al}_{2} \mathrm{O}_{3}$ grains plus an outflow $(50 \%$ $\mathrm{Ca}_{2} \mathrm{Mg}_{0.5} \mathrm{Al}_{2} \mathrm{Si}_{1.5} \mathrm{O}_{7}$ and $50 \%$ metallic iron) in which the mass-loss rate is $4 \times 10^{-10} M_{\odot}$ year $^{-1}$ from $50 \mathrm{AU}$ to $500 \mathrm{AU}$ and $2 \times 10^{-10} M_{\odot} \mathrm{year}^{-1}$ from $500 \mathrm{AU}$ to $1800 \mathrm{AU}$. The stellar input black-body spectrum is shown by a dashed light blue line. For comparison the best model from Paper $\mathrm{I}$ is shown by the long dashed green line.

In Fig. 11 we compare the new model for the $\mathrm{CO}$ emission that adopts the new dust model and the jump in the gas-massloss rate from $1.3 \times 10^{-7}$ to $7 \times 10^{-8} M_{\odot}$ year $^{-1}$ at $500 \mathrm{AU}$. The new $\mathrm{CO}$ model reproduces the $\mathrm{CO}$ rotational lines as well as a model with $\mathrm{CO}$ dissociation occurring at $500 \mathrm{AU}$ and thus is in concordance with such a $\dot{M}$ discontinuity at this distance.

\subsection{Recent (dust) mass-loss history of WHya}

The mass-loss history over the past $10^{4}-10^{5}$ years is unveiled by the infrared images obtained with PACS and SPIRE and the infrared astronomical satellite (IRAS, Neugebauer et al. 1984). The image taken by PACS at $70 \mu \mathrm{m}$ has the best spatial 


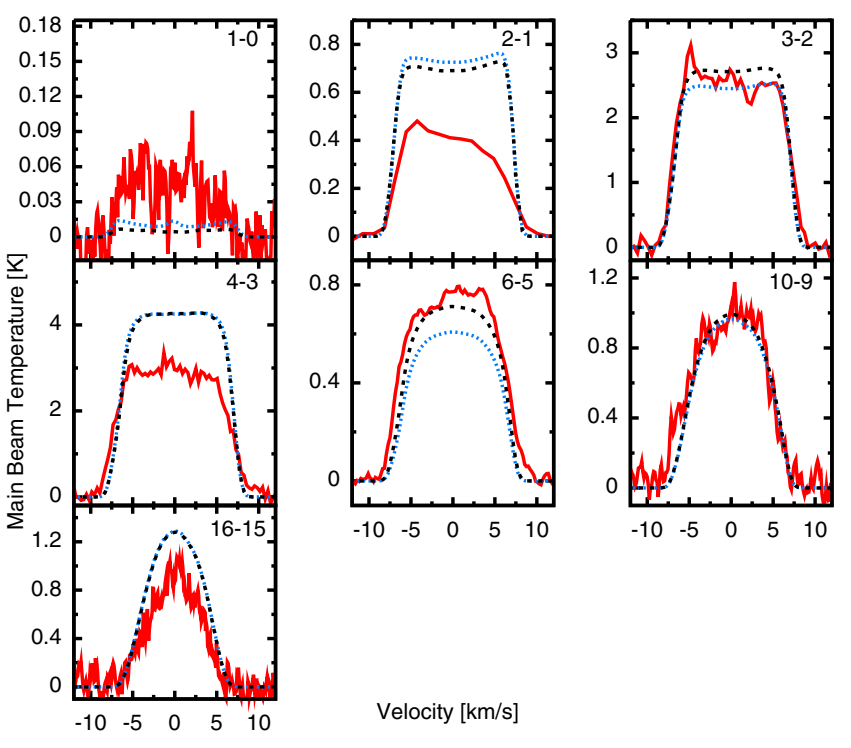

Fig. 11. Comparison of the observed CO rotational emission lines (red solid line) to the model with mass-loss properties as described in the caption of Fig. 10 and a dissociation radius set to the canonical value obtained by Mamon et al. (1988; shown by the blue dotted line). The model obtained in Paper I is shown for comparison (black dashed line).

resolution and, therefore, provides the best constraints. Emission from the extended dust envelope that seems to be produced by a higher mass-loss rate phase is also seen in the infrared ISO spectra (Hawkins 1990). This emission is only produced by cold $(T<70 \mathrm{~K})$ dust and affects the flux measured at the relatively long wavelengths $(\lambda>45 \mu \mathrm{m})$ of the LWS spectral range; it has no impact on the dust emission in the inner wind.

\subsubsection{Infrared images}

The $70 \mu \mathrm{m}$ PACS brightness distribution is rather unusual for an oxygen-rich AGB star (Cox et al. 2012). These images show excess dust emission produced between $30^{\prime \prime}$ and $100^{\prime \prime}$ that cannot be understood in terms of a constant mass-loss wind. In Fig. 9 we show the angle-averaged radial profile of the observed flux density at $70 \mu \mathrm{m}$. The PACS image shows the envelope to have an ellipsoidal shape when projected on the sky. This causes the radial profile averaged over all directions to have a shallower slope from around $75^{\prime \prime}$ on than radial profiles computed for a narrow range of directions. The difference in continuum level of about $20 \%$ between PACS and ISO at $70 \mu \mathrm{m}$ is an effect of the significantly smaller field of view of the PACS spectrometer compared to that of ISO LWS.

On a larger scale, $100 \mu \mathrm{m}$ maps obtained with IRAS show a very extended dust shell, $\approx 30^{\prime}$ in diameter, which requires a high mass-loss rate to explain it (Hawkins 1990). Together, the PACS and IRAS infrared maps show that the dust mass-loss rate of W Hya has not been constant in the past $10^{4}$ to $10^{5}$ years.

\subsubsection{Model for the recent mass-loss history}

To model this extended emission, we used the inner wind dust composition and applied variations in the dust mass-loss rate. Since the PACS image shows an elliptical dust envelope and our model assumes a spherically symmetric wind, we have measured the radial brightness profile in a narrow range of directions (from $130^{\circ}$ to $170^{\circ}$ measured from north to east) and used that to constrain our model. Measuring the radial profile in a different

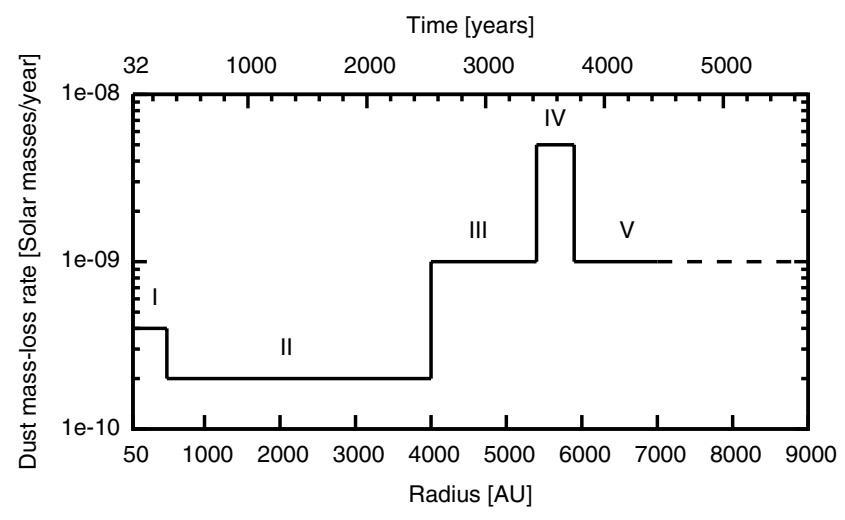

Fig. 12. Schematic dust mass-loss history of W Hya. The mass-loss rate shown by the dashed line could not be constrained by our model. The timescales given along the upper $x$-axis is calculated assuming a constant expansion velocity of $7.5 \mathrm{~km} \mathrm{~s}^{-1}$.

direction produces a similar curve with the peak seen at $75^{\prime \prime}$ in Fig. 9 shifted to larger distances from the star. The chosen direction corresponds to the direction in which the radius of the observed circumstellar shell is the smallest. The asymmetry seen in the PACS image could, in principle, be due to a direction dependent expansion velocity, but that is not considered in our modelling. The largest radius measured is larger by about $25 \%$ than the one modelled by us. A difference of $25 \%$ in radius translates into a difference in expansion velocity of also $25 \%$.

The dotted purple line in Fig. 9 shows the fit to the profile composed of a combination of the constant mass-loss rate epochs shown in Fig. 12. The mass-loss history and the constraints motivating this model are given in Table 2 . The values given assume the present-day expansion velocity of $7.5 \mathrm{~km} \mathrm{~s}^{-1}$, measured from the gas lines. If the gas-to-dust ratio remained constant (at 325), the dust mass-loss rates of phases II, III, and IV would correspond to $6.5 \times 10^{-8}, 3.9 \times 10^{-7}$, and $2.0 \times$ $10^{-6} M_{\odot}$ year $^{-1}$ of gas. The exact values of the derived dust mass-loss rates depend on the model for the present-day dust mass-loss. If the composition of the dust producing emission at $\approx 5000$ AU is not significantly different from that of the inner silicate envelope ( $r \gtrsim 50 \mathrm{AU})$, the derived ratio between the high and low mass-loss rate phases should be accurate even if our inner-wind model were not. If the dust composition and/or expansion velocity changes significantly between these two components, our results probably underestimate the mass-loss rate for the phase of stronger mass loss. That is because, first, the opacity of $\mathrm{Ca}_{2} \mathrm{Mg}_{0.5} \mathrm{Al}_{2} \mathrm{Si}_{1.5} \mathrm{O}_{7}$ at $70 \mu \mathrm{m}$ is higher than that of other silicates, so more mass of other silicate species at the same temperature would be required to produce the same emission at these wavelengths. And, second, the expansion velocity tends to increase with increasing mass-loss rate (see, e.g., Olofsson et al. 2002), which means that the present value of $7.5 \mathrm{~km} \mathrm{~s}^{-1}$ would probably be lower than the expansion velocity during a phase with higher mass-loss rate.

\section{Discussion}

We have modelled the dust envelope of W Hya using two components: a GBDS from which the $\mathrm{Al}_{2} \mathrm{O}_{3}$ emission originates and an outflow that contains silicates that are in thermal contact with metallic iron. Overall, the best-fit model (see Fig. 10) provides a good fit to the infrared emission, although the position of the silicate peak at $9.7 \mu \mathrm{m}$ is not well reproduced. Our model does not require a super-solar aluminum abundance that is required if 
Table 2. Mass-loss history of W Hya.

\begin{tabular}{lrrl}
\hline \hline Phase & $\begin{array}{c}\dot{M}_{\text {dust }} \\
{\left[M_{\odot} \text { year }^{-1}\right]}\end{array}$ & $\begin{array}{r}R_{\text {in }} \\
{[\mathrm{AU}]}\end{array}$ & Constraint \\
\hline I & $4.0 \times 10^{-10}$ & 50 & ISO spectrum \\
II & $2.0 \times 10^{-10}$ & 500 & $70 \mu$ m profile peak + CO model \\
III & $1.2 \times 10^{-9}$ & 4000 & $70 \mu$ m profile from 20 to $60^{\prime \prime}$ \\
IV & $6.0 \times 10^{-9}$ & 5400 & $70 \mu$ m profile beyond $60^{\prime \prime}$ \\
V & $1.2 \times 10^{-9}$ & 5900 & $70 \mu$ m profile beyond $60^{\prime \prime}$ \\
\hline
\end{tabular}

the $\mathrm{Al}_{2} \mathrm{O}_{3}$ grains that cause the emission at $12 \mu \mathrm{m}$ are part of the outflow. The silicate emission originates from beyond $50 \mathrm{AU}$ and best fits the spectrum if silicate grains that contain $\mathrm{Al}$ and $\mathrm{Ca}$ are used. This again introduces a super-solar abundance problem but we argue that this may be a spurious problem. We now discuss the empirically derived envelope structure in the context of wind dynamics, the gas- and solid-phase silicon budget and the elemental abundances of $\mathrm{Al}$ and $\mathrm{Ca}$, and the expected crystallinity of the dust grains.

\subsection{Scattering agents and wind driving in oxygen-rich AGB stars with a low mass-loss rate}

The spectrum of W Hya is well-explained by a GBDS of amorphous $\mathrm{Al}_{2} \mathrm{O}_{3}$ that produces most of the thermal emission seen at $12 \mu \mathrm{m}$. For the fit to the scattered light fractions our models can not distinguish between the two species that have been suggested, namely silicates or $\mathrm{Al}_{2} \mathrm{O}_{3}$. Although $\mathrm{Al}_{2} \mathrm{O}_{3}$ is able to explain the scattered light and thermal emission simultaneously, wind-driving models suggest that a population of pure amorphous $\mathrm{Al}_{2} \mathrm{O}_{3}$ grains provides insufficient opacity to initiate a wind. Silicates, however, are much more effective in initiating the outflow because of the higher abundance of silicon (Bladh $\&$ Höfner 2012). To illustrate this: condensation of $35 \%$ of the available silicon in a solar composition gas into large grains of a typical silicate glass increases the scattering opacity by an order of magnitude relative to dust that is composed of fully condensed large amorphous $\mathrm{Al}_{2} \mathrm{O}_{3}$ grains alone. If large amorphous $\mathrm{Al}_{2} \mathrm{O}_{3}$ grains are lacking in the GBDS the $12 \mu \mathrm{m}$ emission is produced mainly or exclusively by small amorphous $\mathrm{Al}_{2} \mathrm{O}_{3}$ grains.

The gas mass that should be present in the GBDS to account for the amorphous $\mathrm{Al}_{2} \mathrm{O}_{3}$ emission is $\sim 10^{-5} M_{\odot}$. Given the mass-loss rate of W Hya of $1.3 \times 10^{-7} M_{\odot}$ year $^{-1}$, this implies that only a small fraction (a few percent) of the GBDS is expelled per pulsation cycle of about a year. The gas mass in the GBDS that is required to produce the scattered light - assuming it is due to $35 \%$ silicon condensing in large $\mathrm{Mg}_{2} \mathrm{SiO}_{4}$ grains is $\sim 2.3 \times 10^{-7} M_{\odot}$. This mass is more than an order of magnitude lower than the corresponding mass for amorphous $\mathrm{Al}_{2} \mathrm{O}_{3}$, and is of about the same magnitude as the total mass that is lost per year. If the silicates are responsible for the scattering and condense on the amorphous $\mathrm{Al}_{2} \mathrm{O}_{3}$ seeds, condensation of this material should occur over a small radial interval of the GBDS. This is because the composite grains must be cold not to produce silicate emission and, therefore, would also not produce any amorphous $\mathrm{Al}_{2} \mathrm{O}_{3}$ emission. Because of this and as a result of the low abundance of aluminum, a relatively larger fraction of the GBDS volume in which warm amorphous $\mathrm{Al}_{2} \mathrm{O}_{3}$ grains exist is needed to reproduce the observed emission. Models of outflows driven by large $\mathrm{Mg}_{2} \mathrm{SiO}_{4}$ particles indeed show very rapid growth of these grains in the onset region of the flow (see, e.g., Höfner 2008).

Alternatively, the grains responsible for the scattering could be aggregates of different oxides including species other than

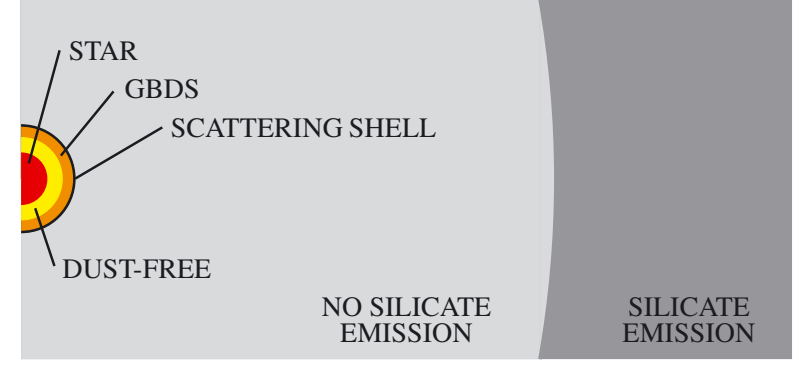

Fig. 13. Schematic view of the envelope of WHya. The radii of the different structures are given to scale. The radius of the star and the scattering shell are 1.8 AU and 3.6 AU. The inner radius of the GBDS is not constrained by our model and is assumed to be $3.1 \mathrm{AU}\left(1.7 R_{\star}\right)$ for the small amorphous $\mathrm{Al}_{2} \mathrm{O}_{3}$ grains. The inner radius of the silicate envelope shown is the lower limit of $40 \mathrm{AU}$ given by Zhao-Geisler et al. (2011).

silicates and $\mathrm{Al}_{2} \mathrm{O}_{3}$ - such as calcium or magnesium oxides but we have not explored this possibility.

\subsection{Structure of the dust envelope of WHya}

A schematic view of the model for the envelope of W Hya is shown in Fig. 13. Most of the amorphous $\mathrm{Al}_{2} \mathrm{O}_{3}$ emission can be accounted for by grains in the GBDS. The thickness of this GBDS is difficult to assess as its inner radius depends on the poorly constrained near-infrared optical constants of amorphous $\mathrm{Al}_{2} \mathrm{O}_{3}$. At about $2 R_{\star}$ grains of $0.3 \mu \mathrm{m}$ are present to account for the scattered light fractions observed. In our models, these grains can be either amorphous $\mathrm{Al}_{2} \mathrm{O}_{3}$ or iron-free silicates. If these scatterers are silicates, it is likely that the amorphous $\mathrm{Al}_{2} \mathrm{O}_{3}$ grains serve as seeds for silicate condensation (Kozasa \& Sogawa 1997b). If silicates exist so close to the star, they have to be translucent in the near-infrared and remain cold such as not to produce significant emission. Combined with the broadness of the brightness peak in the PACS $70 \mu \mathrm{m}$ image, this implies that the bulk of silicate emission at $9.7 \mu \mathrm{m}$ has to come from an envelope with an inner radius of $50 \mathrm{AU}$. The gas temperature at this distance is $\sim 350 \mathrm{~K}$ (Khouri et al. 2014b).

If the silicates are the scatterers and are present near the star without producing significant emission, there would have to be a physical process that increases their temperature beyond $50 \mathrm{AU}$. Such a process could be a change in their lattice structure, which increases the absorption in the near-infrared, or the establishing of contact with a more opaque dust species, for instance small iron grains - creating a silicate with iron inclusions. Alternatively, if the scattering is caused by $\mathrm{Al}_{2} \mathrm{O}_{3}$, or grains composed of a combination of other oxides, the inner radius of the silicate emission envelope could be set by silicate condensation. It is not clear, however, why any of the processes mentioned would occur at such large distances. This interpretation is based on the assumption of a steady-state wind model for the inner regions, $r<1000 \mathrm{AU}$. The observed radial dependency of the dust-envelope parameters of W Hya could potentially also be the result of variable conditions at the wind acceleration region, but this explanation seems less likely (see Sect. 4.5).

\subsection{Bringing the dust and gas models together: elemental abundances in the inner wind.}

The abundances of the elemental species that are required to reproduce the thermal emission from the wind of W Hya are 4.8 times the solar-composition value for calcium, 0.06 for magnesium, 3.5 for aluminum, 0.24 for silicon, 0.07 for oxygen, and 
1.15 for iron. If the opacity of the metallic iron grains is computed using a CDE distribution of sizes, a factor of three less iron is needed. In what follows, we discuss the abundances of the dust species in more detail and offer potential solutions to the apparent super-solar abundances of calcium and aluminum.

\subsubsection{Silicon abundance}

Khouri et al. (2014b) modelled the gas-phase SiO emission and found that between 15 and $50 \%$ of the silicon atoms are expected to be depleted from the gas-phase. When considered together with our dust model this accounts for all the silicon expected for a solar composition wind.

However, since the calcium-bearing silicate that we adopted $\left(\mathrm{Ca}_{2} \mathrm{Mg}_{0.5} \mathrm{Al}_{2} \mathrm{Si}_{1.5} \mathrm{O}_{7}\right)$ does not provide a perfect fit to the $9.7 \mu \mathrm{m}$ silicate peak, the constraints on the solid-phase silicon abundance are not stringent. To assess this problem, we have compared models of other silicates that provide a better fit to the $9.7 \mu \mathrm{m}$ peak silicate feature to the observed spectrum. These dust species provide a poorer fit to the overall spectrum, however, as they reproduce neither the $18 \mu \mathrm{m}$ silicate peak nor the $30 \mu \mathrm{m}$ feature. We have chosen to fit the flux ratio between the $9.7 \mu \mathrm{m}$ silicate peak and the trough at 11.9 and $14.5 \mu \mathrm{m}$. The trough flux is sensitive to the amount of iron in thermal contact with the silicates, which needs to be $50 \%$ per mass, typically, in our models. The best fits are always achieved with in between $30 \%$ and $40 \%$ of the silicon available from the gas phase. Therefore, the amount of silicon needed in the dust agrees with the depletion fraction reported by Khouri et al. (2014b) independent of the exact composition of the silicate. This is in virtue of the strength of the $9.7 \mu$ m peak correlating well with the $\mathrm{SiO}_{2}$ content of the given silicate species (e.g. Koike \& Hasegawa 1987).

\subsubsection{Aluminum and calcium abundances}

In our model, aluminum is condensed in amorphous $\mathrm{Al}_{2} \mathrm{O}_{3}$ grains in the GBDS and also in silicates present in the wind. We do not require amorphous $\mathrm{Al}_{2} \mathrm{O}_{3}$ to be present in the wind but a significant amount in terms of aluminum abundance could be driven out without producing a strong spectral signature. We considered only the outflowing aluminum-bearing grains in the aluminum budget, as the grains that form in the GBDS form from a gravitationally bound gas mass that is not independently constrained by our models. Nonetheless, our dust model requires 3.5 times more aluminum than what is available from the gas phase. Unfortunately, we do not have aluminum-free calciumbearing silicates in our dataset of optical constants, so we cannot directly probe the impact of the aluminum content on the optical properties of $\mathrm{Ca}_{2} \mathrm{Mg}_{0.5} \mathrm{Al}_{2} \mathrm{Si}_{1.5} \mathrm{O}_{7}$. Gervais et al. (1987) studied the influence of aluminum content on silicate emission features and found it to have a minor impact on the strongest ressonances. This suggests that the over-abundance of aluminum found by us may be spurious and that an equally good fit to the $\mathrm{Al}_{2} \mathrm{O}_{3}-$ subtracted residual spectrum could be achieved with a silicate with a lower aluminum content.

The required super-solar abundance of calcium is somewhat more intriguing, as this element seems to have a significant impact on the strengths and shapes of the silicate features. Particularly the $30 \mu \mathrm{m}$ feature is linked to the presence of this element. Like in the case of aluminum, however, we do not have a library of optical constants for silicate species with different amounts of calcium content, which prevents us from assessing its relation to the $30 \mu \mathrm{m}$ feature.

We identify three potential solutions to the problem of the super-solar abundance of aluminum and calcium in the dust found by us. The first option is that silicates that contain smaller amounts of calcium and aluminum are responsible for the thermal emission. Speck et al. (2011) presented measurements of silicates with a range of compositions and calcium and aluminum content. One of the species studied by them, named basalt in their work, has calcium-to-silicon and aluminum-to-silicon ratios very similar to those expected for $20 \%$ silicon condensation and full calcium and aluminum condensation. The absorbance spectrum of this species shown in Fig. 4 of their paper peaks at $10 \mu \mathrm{m}$, which is closer to the observed peak position for W Hya than that of $\mathrm{Ca}_{2} \mathrm{Mg}_{0.5} \mathrm{Al}_{2} \mathrm{Si}_{1.5} \mathrm{O}_{7}$. The $18 \mu \mathrm{m}$ peak of this species is also in accordance with the observed spectrum of W Hya. Unfortunately for our purposes, the authors focused on analysing the $10 \mu \mathrm{m}$ and $18 \mu \mathrm{m}$ region and we have no information on the spectra of this species at $30 \mu \mathrm{m}$. Measurements of optical data for silicates with these characteristics are needed to determine whether the spectrum of W Hya can be reproduced by such a species.

A second possibility to explain the apparent super-solar abundance of $\mathrm{Al}$ and $\mathrm{Ca}$ is that some of the features fitted by $\mathrm{Ca}_{2} \mathrm{Mg}_{0.5} \mathrm{Al}_{2} \mathrm{Si}_{1.5} \mathrm{O}_{7}$, such as those at $20 \mu \mathrm{m}$ and $30 \mu \mathrm{m}$, are partially produced in the GBDS, similarly to $\mathrm{Al}_{2} \mathrm{O}_{3}$. In that case the abundance problem would not exist. This can be the case if other oxides are able to condense in the GBDS. Calcium is indeed expected to be one of the first elements to react with $\mathrm{Al}_{2} \mathrm{O}_{3}$ from condensation calculations (Grossman \& Larimer 1974). If calcium reacts with and is incorporated into the $\mathrm{Al}_{2} \mathrm{O}_{3}$ grains before the onset of the wind, other features in the spectrum could also be produced in the extended atmosphere. Furthermore, the addition of calcium to the dust grains in the GBDS could help driving the wind. However, the increase in the dust-to-gas ratio due to calcium condensation would only be of about a factor of two.

Finally, calcium and aluminum could be overabundant in the wind if these atoms are included in the dust grains before the wind is fully driven and if only a fraction of the gas is driven out by these grains. Although this might be the case, this scenario seems unlikely as the dust grains should be well coupled to the gas at the densities expected in the region where the flow is initiated.

\subsection{Lattice structure of the grains in the GBDS}

The grains in the GBDS must form from high-temperature gas and are heated by the stellar radiation field to temperatures that may differ from that of the gas. Being subjected to high enough temperatures with respect to the glass temperature of the given dust species will cause the dust grains to become crystalline. Whether the lattice structures of the dust grains are amorphous or crystalline is directly connected to the features produced by them in the infrared spectrum. In particular, our model requires that amorphous $\mathrm{Al}_{2} \mathrm{O}_{3}$ grains exist close to the star $\left(\sim 2 R_{\star}\right)$ to reproduce the ISO spectrum. As we obtain the grain temperature as a function of radius, we can speculate what the lattice structure of $\mathrm{Al}_{2} \mathrm{O}_{3}$ and silicate grains in the GBDS would be and test if that is consistent with our assumptions.

\subsection{1. $\mathrm{Al}_{2} \mathrm{O}_{3}$ grains}

The physical conditions in AGB outflows favour the condensation of amorphous $\mathrm{Al}_{2} \mathrm{O}_{3}$ grains (e.g. Dell'Agli et al. 2014). In our calculations the temperature of the grains in the GBDS is found to be between $1500 \mathrm{~K}$ and $1600 \mathrm{~K}$ for large grains and between $1200 \mathrm{~K}$ and $1300 \mathrm{~K}$ for small grains. Begemann et al. (1997) found that at $1300 \mathrm{~K} \mathrm{Al}_{2} \mathrm{O}_{3}$ grains are crystalline, while Levin et al. (1998) reported that the crystalline component only 
dominates at higher temperatures $(\sim 1450 \mathrm{~K})$. The optical data at short wavelengths for $\mathrm{Al}_{2} \mathrm{O}_{3}$ are quite uncertain, which translates into an uncertainty in the calculated temperature of the amorphous $\mathrm{Al}_{2} \mathrm{O}_{3}$ grains. For instance, a lower opacity in the near-infrared would cause the grains to be cooler. If we consider the size and location of the amorphous $\mathrm{Al}_{2} \mathrm{O}_{3}$ shell and maintain the amounts of grains, the emission produced is proportional to the Planck function, since the emitting medium is optically thin. Therefore, a lower temperature of 1400 or $1300 \mathrm{~K}$ for the large grains would correspond to 10 or $20 \%$ less emission, respectively, and the amorphous $\mathrm{Al}_{2} \mathrm{O}_{3}$ from the shell could still dominate the emission at $12 \mu \mathrm{m}$.

It is also possible, and quite likely, that crystalline and amorphous $\mathrm{Al}_{2} \mathrm{O}_{3}$ co-exist in the envelope of oxygen-rich AGB stars. In fact, grains consisting of pure crystalline $\mathrm{Al}_{2} \mathrm{O}_{3}$ (Zeidler et al. 2013) and those consisting of a crystalline $\mathrm{Al}_{2} \mathrm{O}_{3}$ core and a silicate mantle (Kozasa \& Sogawa 1997a) have been proposed as the carriers of the unidentified $13 \mu \mathrm{m}$ feature. We tried to fit the $13 \mu \mathrm{m}$ feature of $\mathrm{W}$ Hya with pure crystalline $\mathrm{Al}_{2} \mathrm{O}_{3}$ grains in the GBDS using the optical constant measured by Zeidler et al. (2013). We can only obtain a good fit by considering spherical (rather than DHS) particles and the optical constants for crystalline $\mathrm{Al}_{2} \mathrm{O}_{3}$ grains at $973 \mathrm{~K}$. Our models show that the mass abundance of crystalline $\mathrm{Al}_{2} \mathrm{O}_{3}$ grains compared to the amorphous ones would be very small, around $5 \%$ by mass. We note that Takigawa et al. (2014) found a high fraction of crystalline pre-solar $\mathrm{Al}_{2} \mathrm{O}_{3}$ grains. The authors measured five out of nine studied pre-solar $\mathrm{Al}_{2} \mathrm{O}_{3}$ grains, which were probably produced in the ejecta of a low-mass AGB star. The derived degree of crystallinity is considerably higher than that inferred from the model presented in this work.

\subsubsection{Silicate grains}

We can speculate on what would be the lattice structure of silicate solids if they condensed on a warm $\mathrm{Al}_{2} \mathrm{O}_{3}$ core in the GBDS. If a silicate solid is heated to a temperature above its glass temperature $(\sim 1000 \mathrm{~K})$, it will crystallize. Large pure $\mathrm{Mg}_{2} \mathrm{SiO}_{4}$ grains have a temperature of $700 \mathrm{~K}$ at $2.0 R_{\star}$; a population of small pure amorphous $\mathrm{Al}_{2} \mathrm{O}_{3}$ grains at the same distance is heated to about $1300 \mathrm{~K}$. The temperature of a composite grain will obviously depend on the relative fraction of each species. If a low mass of $\mathrm{Mg}_{2} \mathrm{SiO}_{4}$ condenses onto the surface of an amorphous $\mathrm{Al}_{2} \mathrm{O}_{3}$ core the $\mathrm{Mg}_{2} \mathrm{SiO}_{4}$ solid is expected to be crystalline because the temperature of the composite would rise above the glass temperature for silicates. However, if $35 \%$ silicon condensation and full aluminum condensation is assumed, the silicate grain mass would be approximately 11.5 times that of amorphous $\mathrm{Al}_{2} \mathrm{O}_{3}$ and the composite grains would probably remain cold and undetectable. A rough calculation suggests that when the mass of $\mathrm{Mg}_{2} \mathrm{SiO}_{4}$ and amorphous $\mathrm{Al}_{2} \mathrm{O}_{3}$ are comparable, the temperature of the composite grain would be about $1000 \mathrm{~K}$ at $2.0 R_{\star}$, that is close to the glass temperature for silicates. This means that only roughly the first $10 \%$ of silicate material to condense onto an amorphous $\mathrm{Al}_{2} \mathrm{O}_{3}$ grain in the GBDS would yield a crystalline solid, while continued silicate condensation would make the grain amorphous.

\subsection{Expansion velocity profile of the wind of WHya}

In our best-fit model, the silicate emission is produced in an envelope with inner radius of $50 \mathrm{AU}$. In order for these grains to be visible, large amounts of metallic iron must be placed in thermal contact with the silicate grains. We can connect this finding with the gas model from Paper I. In that work, the wind of
W Hya was found to slowly accelerate up to about $5.5 \mathrm{~km} \mathrm{~s}^{-1}$ and then to quickly accelerate to the final expansion velocity of $7.5 \mathrm{~km} \mathrm{~s}^{-1}$. Such an additional acceleration requires extra opacity to add momentum to the (supersonic) flow. In a dust-driven outflow, changes in the dust properties could in principle provide such additional momentum. We speculate that the late condensation of metallic iron on the silicate grains might provide the extra opacity needed for an increase in the wind speed to its final value.

This interpretation is based on a steady-state picture for the inner wind ( $r \lesssim 1000 \mathrm{AU})$. An alternative explanation is that the conditions at the wind-acceleration region have changed about 30 years ago, causing the dust composition to change from silicate-rich to silicate-poor and the wind expansion velocity to decrease. However, we do not see signs of significant mass-loss rate variations in the acceleration zone, as one might expect if the local conditions had significantly changed. In addition, the expansion velocity measured using different diagnostics has been shown to increase steadily outwards (see, e.g., Muller et al. 2008; Szymczak et al. 1998). Moreover, if the material at $r>50 \mathrm{AU}$ and now expanding at $7.5 \mathrm{~km} \mathrm{~s}^{-1}$ had been ejected with a higher velocity from the start than material inwards, one would expect to see a gap (of about $15 \mathrm{AU}$ ) between these two wind components. There is no support for such a gap in the data at hand. Although we are not able to rule out this alternative explanation completely, we consider it unlikely.

\subsection{Recent mass-loss history of W Hya}

We find that W Hya has undergone abrupt changes of more than an order of magnitude in its dust mass-loss rate in the past few thousand years. Hawkins (1990) reported observations carried out with IRAS that reveal a dust shell of $1 \times 10^{-4} M_{\odot}$ extending to about $15^{\prime}$. If the same expansion velocity is considered, it would take the dust grains forty-five thousand years to reach such distances from the star and, therefore, the average dust mass-loss rate in that period would be $2 \times 10^{-9} M_{\odot}$ year $^{-1}$. This value is compatible with the mass-loss rate derived by us for the phases with high mass-loss rates (phases II, IV and V, given in Table 2), rather than to the more recent period of low mass-loss rate (phases I and II, given in Table 2). Therefore, if the mass loss fluctuates between a low and high mode, the high mode must be the norm. W Hya might have recently undergone a change in mass-loss rate that had not occurred in the past fifty thousand years.

Although thin shells produced by similar mass-loss rate discontinuities are found somewhat frequently around carbon-rich AGB stars (e.g. Olofsson et al. 1990, 1996), the narrow massloss rate peak seen between 5400 and 5900 AU in the PACS images of W Hya is unusual for oxygen-rich AGB stars (Cox et al. 2012). The shells around carbon-stars are thought to form as a result of a strong increase in mass-loss rate during a thermal-pulse (Schroeder et al. 1998; Mattsson et al. 2007). It is not known why these detached shells are not observed around oxygen-rich AGB stars, and W Hya is unique in that sense. The zero-age-mainsequence mass of W Hya was determined to be $1.5 \pm 0.2 M_{\odot}$ (Khouri et al. 2014b), which places this object around the limit for becoming a carbon-rich star (Wallerstein \& Knapp 1998). However, models for the formation of detached shells require the interaction of a faster wind with a previously slower wind (e.g. Schöier et al. 2005), and that is not expected to be directly connected to stellar mass, but rather to changes in the wind properties during the thermal pulse. Hence, it is currently not clear whether the peak in the mass-loss rate of W Hya is due to a 
thermal pulse or whether the star can be seen as an oxygen-rich analogue to carbon stars with detached shells.

\section{Summary}

We presented a model for the dust envelope of W Hya that simultaneously considers different observables of the dust envelope of this star: the scattered light fractions (Norris et al. 2012), the ISO spectrum (Justtanont et al. 2004; Sloan et al. 2003), the inner radius of the silicate emission shell (Zhao-Geisler et al. 2011), the elemental abundances in the wind (Paper I, Khouri et al. 2014b), and the PACS $70 \mu \mathrm{m}$ image (Cox et al. 2012).

Our model consists of a gravitationally bound dust shell combined with an outflow. An MRN distribution of amorphous $\mathrm{Al}_{2} \mathrm{O}_{3}$ particles in the GBDS fits both the infrared excess emission and the scattered light fractions. However, large translucent silicates that show no spectral signature at $9.7 \mu \mathrm{m}$, but that are able to reproduced the scattered light fractions, can exist in the GBDS and wind, implying that we are unable to determine the nature of the scattering agents. Silicate grains are preferred, however, based on wind-driving models because of the low abundance of aluminum (Höfner 2008; Bladh \& Höfner 2012). Independent of the nature of the grains that drive the outflow, spectral emission from the silicate grains arises from distances beyond $50 \mathrm{AU}$ from the star. This emission may arise when iron inclusions are incorporated into the silicates heating up the grains. The silicon content that is in the dust is found to be consistent with the amount of silicon atoms that disappears from the gas phase, as extracted from $\mathrm{SiO}$ line emission (Khouri et al. 2014b). We find evidence that calcium is a constituent of the dust in the wind of WHya. It is difficult, however, to quantify the calcium content of the dust because we only have optical constants available for three calcium-bearing species. Although our best fit seems to suggest a super-solar $\mathrm{Ca}$ abundance in the outflow, optical data over a broad wavelength range of silicates with lower calcium content might result in fits with a solar calcium abundance. Emission from calcium-bearing dust species might also originate from the GBDS, as long as there is no strong contribution in the MIDI wavelength range.

The mass-loss rate of W Hya seems to have been at the present level for about 300 years. At times before this, we see a phase when the mass-loss was lower by a factor of roughly two, which is traced by both the dust envelope and the $\mathrm{CO}$ gas emission. The mass-loss rate remained at this level for about 2500 years and produced dust now located in the envelope between 500 and 4000 AU. This phase of lower mass loss was preceded by one where $\mathrm{W}$ Hya lost mass at a rate at least three times higher than at present, with a localised peak in which the rate was even twenty times higher and which ended about 3400 years ago and lasted for about 300 years.

Acknowledgements. The authors thank the anonymous referee for the careful reading of the paper and the suggestions that helped improve the quality and clarity of the text. PACS has been developed by a consortium of institutes led by MPE (Germany) and including UVIE (Austria); KUL, CSL, IMEC (Belgium); CEA, OAMP (France); MPIA (Germany); IFSI, OAP/AOT, OAA/CAISMI, LENS, SISSA (Italy); IAC (Spain). This development has been supported by the funding agencies BMVIT (Austria), ESA-PRODEX (Belgium), CEA/CNES (France), DLR (Germany), ASI (Italy), and CICYT/MCYT (Spain). T.Kh. gratefully acknowledges the support from NWO grant 614.000.903. N.L.J.C. acknowledges support from the Belgian Federal Science Policy Office via the PRODEX Programme of ESA.

\section{Appendix A: Silicate emission}

Table A.1. Diagnostic parameters of the silicate dust species tested by us in the fitting procedure.

\begin{tabular}{|c|c|c|c|c|c|c|}
\hline Species & Ref. & $\begin{array}{c}\kappa_{9.7 \mu \mathrm{m}}^{\max } \\
{\left[\mathrm{cm}^{2} / \mathrm{g}\right]}\end{array}$ & $\begin{array}{c}\lambda \kappa_{9.7 \mu \mathrm{m}}^{\max } \\
{[\mu \mathrm{m}]}\end{array}$ & $\begin{array}{c}\kappa_{18 \mu \mathrm{m}}^{\max } \\
{\left[\mathrm{cm}^{2} / \mathrm{g}\right]}\end{array}$ & $\begin{array}{c}\lambda \kappa_{18 \mu \mathrm{m}}^{\max } \\
{[\mu \mathrm{m}]}\end{array}$ & $\kappa_{18 \mu \mathrm{m}}^{\max } / \kappa_{9.7 \mu \mathrm{m}}^{\max }$ \\
\hline $\mathrm{Ca}_{2} \mathrm{Al}_{2} \mathrm{SiO}_{7}$ & 1 & 3210 & 10.4 & 1270 & 20.7 & 0.40 \\
\hline $\mathrm{Ca}_{2} \mathrm{Mg}_{0.5} \mathrm{Al}_{2} \mathrm{Si}_{1.5} \mathrm{O}_{7}$ & 1 & 3920 & 10.3 & 1450 & 19.4 & 0.37 \\
\hline $\mathrm{Mg}_{0.4} \mathrm{Fe}_{0.6} \mathrm{SiO}_{3}$ & 2 & 3050 & 9.5 & 1680 & 20.9 & 0.55 \\
\hline $\mathrm{Mg}_{0.5} \mathrm{Fe}_{0.43} \mathrm{Ca}_{0.03} \mathrm{Al}_{0.04} \mathrm{SiO}_{3}$ & 3 & 3640 & 9.7 & 1670 & 18.9 & 0.46 \\
\hline $\mathrm{Mg}_{0.5} \mathrm{Fe}_{0.5} \mathrm{SiO}_{3}$ & 2 & 3370 & 9.5 & 1720 & 19.3 & 0.51 \\
\hline $\mathrm{Mg}_{0.7} \mathrm{Fe}_{0.3} \mathrm{SiO}_{3}$ & 2 & 3940 & 9.6 & 1970 & 18.6 & 0.50 \\
\hline $\mathrm{Mg}_{0.7} \mathrm{SiO}_{2.7}$ & 4 & 2720 & 9.3 & 1530 & 20.9 & 0.56 \\
\hline $\mathrm{Mg}_{0.8} \mathrm{Fe}_{0.2} \mathrm{SiO}_{3}$ & 2 & 4240 & 9.7 & 2000 & 18.6 & 0.47 \\
\hline $\mathrm{Mg}_{0.8} \mathrm{Fe}_{1.2} \mathrm{SiO}_{4}$ & 2 & 2610 & 10.2 & 1800 & 18.0 & 0.69 \\
\hline $\mathrm{Mg}_{1.5} \mathrm{Fe}_{1.5} \mathrm{AlSi}_{3} \mathrm{O}_{10.5}$ & 1 & 3790 & 10.2 & 1700 & 18.8 & 0.45 \\
\hline $\mathrm{Mg}_{1.5} \mathrm{SiO}_{3.5}$ & 4 & 2460 & 9.8 & 1440 & 18.9 & 0.59 \\
\hline $\mathrm{Mg}_{2.4} \mathrm{SiO}_{4.4}$ & 4 & 1590 & 10.2 & 1370 & 17.8 & 0.86 \\
\hline $\mathrm{Mg}_{2} \mathrm{AlSi}_{2} \mathrm{O}_{7.5}$ & 1 & 4480 & 10.0 & 1740 & 18.7 & 0.39 \\
\hline $\mathrm{Mg}_{2} \mathrm{Fe}_{2} \mathrm{AlSi}_{4} \mathrm{O}_{13.5}$ & 1 & 3850 & 10.1 & 1730 & 18.7 & 0.45 \\
\hline $\mathrm{Mg}_{2} \mathrm{SiO}_{4}$ & 4 & 2440 & 9.9 & 1220 & 17.7 & 0.50 \\
\hline $\mathrm{Mg}_{3} \mathrm{AlSi}_{3} \mathrm{O}_{10.5}$ & 1 & 4390 & 10.0 & 1790 & 18.5 & 0.41 \\
\hline $\mathrm{Mg}_{4.5} \mathrm{Fe}_{4.5} \mathrm{AlSi}_{9} \mathrm{O}_{28.5}$ & 1 & 3380 & 10.1 & 1820 & 18.5 & 0.54 \\
\hline $\mathrm{Mg}_{4} \mathrm{AlSi}_{4} \mathrm{O}_{13.5}$ & 1 & 4450 & 9.9 & 1810 & 18.4 & 0.41 \\
\hline $\mathrm{Mg}_{9} \mathrm{AlSi}_{9} \mathrm{O}_{28.5}$ & 1 & 4750 & 9.8 & 1980 & 18.3 & 0.42 \\
\hline $\mathrm{MgFeAlSi}_{2} \mathrm{O}_{7.5}$ & 1 & 3640 & 10.3 & 1590 & 18.6 & 0.44 \\
\hline $\mathrm{MgFeSiO}_{4}$ & 2 & 2700 & 10.1 & 1873 & 18.0 & 0.69 \\
\hline $\mathrm{MgSiO}_{3}$ & 2 & 4910 & 9.6 & 2180 & 18.4 & 0.44 \\
\hline
\end{tabular}

Notes. $\kappa_{9.7 \mu \mathrm{m}}^{\max }, \kappa_{18 \mu \mathrm{m}}^{\max }$ and $\kappa_{18 \mu \mathrm{m}}^{\max } / \kappa_{9.7 \mu \mathrm{m}}^{\max }$ represent the peak value of the opacity in the $9.7 \mu \mathrm{m}$ and $18 \mu \mathrm{m}$ silicate peaks and the ratio between these two values, respectively. $\lambda \kappa_{9.7 \mu \mathrm{m}}^{\max }$ and $\lambda \kappa_{18 \mu \mathrm{m}}^{\max }$ are the wavelengths where the two peaks are found.

References. 1) Mutschke et al. (1998); 2) Dorschner et al. (1995); 3) Jäger et al. (1994); 4) Jäger et al. (2003). 


\section{References}

Asplund, M., Grevesse, N., Sauval, A. J., \& Scott, P. 2009, ARA\&A, 47, 481 Begemann, B., Dorschner, J., Henning, T., et al. 1997, ApJ, 476, 199 Bladh, S., \& Höfner, S. 2012, A\&A, 546, A76

Bladh, S., Höfner, S., Nowotny, W., Aringer, B., \& Eriksson, K. 2013, A\&A, 553, A20

Bohren, C. F., \& Huffman, D. R. 1998, Absorption and Scattering of Light by Small Particles (Wiley-VCH)

Cami, J. 2002, Ph.D. Thesis, University of Amsterdam

Clegg, P. E., Ade, P. A. R., Armand, C., et al. 1996, A\&A, 315, L38

Cox, N. L. J., Kerschbaum, F., van Marle, A.-J., et al. 2012, A\&A, 537, A35

de Graauw, T., Haser, L. N., Beintema, D. A., et al. 1996, A\&A, 315, L49

Dell'Agli, F., García-Hernández, D. A., Rossi, C., et al. 2014, MNRAS, 441, 1115

Dorschner, J., Begemann, B., Henning, T., Jaeger, C., \& Mutschke, H. 1995, A\&A, 300, 503

Fabian, D., Posch, T., Mutschke, H., Kerschbaum, F., \& Dorschner, J. 2001, A\&A, 373, 1125

Gail, H.-P., \& Sedlmayr, E. 1999, A\&A, 347, 594

Gail, H.-P., Wetzel, S., Pucci, A., \& Tamanai, A. 2013, A\&A, 555, A119

Gervais, F., Blin, A., Massiot, D., et al. 1987, J. Non Crystalline Solids, 89, 384

Griffin, M. J., Abergel, A., Abreu, A., et al. 2010, A\&A, 518, L3

Grossman, L., \& Larimer, J. W. 1974, Rev. Geophys. Space Phys., 12, 71

Habing, H. J. 1996, A\&ARv, 7, 97

Habing, H. J., \& Olofsson, H. 2003, Asymptotic Giant Branch Stars (Springer)

Hawkins, G. W. 1990, A\&A, 229, L5

Henning, T., Begemann, B., Mutschke, H., \& Dorschner, J. 1995, A\&AS, 112, 143

Heras, A. M., \& Hony, S. 2005, A\&A, 439, 171

Höfner, S. 2008, A\&A, 491, L1

Ireland, M. J., Tuthill, P. G., Bedding, T. R., Robertson, J. G., \& Jacob, A. P. 2004, MNRAS, 350, 365

Jäger, C., Mutschke, H., Begemann, B., Dorschner, J., \& Henning, T. 1994, A\&A, 292, 641

Jäger, C., Dorschner, J., Mutschke, H., Posch, T., \& Henning, T. 2003, A\&A, 408, 193

Justtanont, K., \& Tielens, A. G. G. M. 1992, ApJ, 389, 400

Justtanont, K., de Jong, T., Tielens, A. G. G. M., Feuchtgruber, H., \& Waters, L. B. F. M. 2004, A\&A, 417, 625

Karovicova, I., Wittkowski, M., Ohnaka, K., et al. 2013, A\&A, 560, A75

Kemper, F., de Koter, A., Waters, L. B. F. M., Bouwman, J., \& Tielens, A. G. G. M. 2002, A\&A, 384, 585

Kessler, M. F., Steinz, J. A., Anderegg, M. E., et al. 1996, A\&A, 315, L27

Khouri, T., de Koter, A., Decin, L., et al. 2014a, A\&A, 561, A5 (Paper I)
Khouri, T., de Koter, A., Decin, L., et al. 2014b, A\&A, 570, A67

Koike, C., \& Hasegawa, H. 1987, Ap\&SS, 134, 361

Koike, C., Kaito, C., Yamamoto, T., et al. 1995, Icarus, 114, 203

Kozasa, T., \& Sogawa, H. 1997a, Ap\&SS, 255, 437

Kozasa, T., \& Sogawa, H. 1997b, Ap\&SS, 251, 165

Levin, I., Gemming, T., \& Brandon, D. G. 1998, Physica Status Solidi Applied Research, 166, 197

Lorenz-Martins, S., \& Pompeia, L. 2000, MNRAS, 315, 856

Mamon, G. A., Glassgold, A. E., \& Huggins, P. J. 1988, ApJ, 328, 797

Mathis, J. S., Rumpl, W., \& Nordsieck, K. H. 1977, ApJ, 217, 425

Mattsson, L., Höfner, S., \& Herwig, F. 2007, A\&A, 470, 339

Min, M., Hovenier, J. W., \& de Koter, A. 2003, A\&A, 404, 35

Min, M., Dullemond, C. P., Dominik, C., de Koter, A., \& Hovenier, J. W. 2009, A\&A, 497, 155

Muller, S., Dinh-V-Trung, He, J.-H., \& Lim, J. 2008, ApJ, 684, L33

Mutschke, H., Begemann, B., Dorschner, J., et al. 1998, A\&A, 333, 188

Neugebauer, G., Habing, H. J., van Duinen, R., et al. 1984, ApJ, 278, L1

Norris, B. R. M., Tuthill, P. G., Ireland, M. J., et al. 2012, Nature, 484, 220

Olofsson, H., Carlstrom, U., Eriksson, K., Gustafsson, B., \& Willson, L. A. 1990, A\&A, 230, L13

Olofsson, H., Bergman, P., Eriksson, K., \& Gustafsson, B. 1996, A\&A, 311, 587

Olofsson, H., González Delgado, D., Kerschbaum, F., \& Schöier, F. L. 2002, A\&A, 391, 1053

Pilbratt, G. L., Riedinger, J. R., Passvogel, T., et al. 2010, A\&A, 518, L1

Poglitsch, A., Waelkens, C., Geis, N., et al. 2010, A\&A, 518, L2

Posch, T., Kerschbaum, F., Mutschke, H., et al. 1999, A\&A, 352, 609

Sacuto, S., Ramstedt, S., Höfner, S., et al. 2013, A\&A, 551, A72

Schöier, F. L., Lindqvist, M., \& Olofsson, H. 2005, A\&A, 436, 633

Schroeder, K.-P., Winters, J. M., Arndt, T. U., \& Sedlmayr, E. 1998, A\&A, 335, L9

Sloan, G. C., Kraemer, K. E., Price, S. D., \& Shipman, R. F. 2003, ApJS, 147, 379

Speck, A. K., Barlow, M. J., Sylvester, R. J., \& Hofmeister, A. M. 2000, A\&AS, 146,437

Speck, A. K., Whittington, A. G., \& Hofmeister, A. M. 2011, ApJ, 740, 93

Szymczak, M., Cohen, R. J., \& Richards, A. M. S. 1998, MNRAS, 297, 1151

Takigawa, A., Tachibana, S., Huss, G. R., et al. 2014, Geochim. Cosmochim. Acta, 124, 309

Verhoelst, T., Decin, L., van Malderen, R., et al. 2006, A\&A, 447, 311

Wallerstein, G., \& Knapp, G. R. 1998, ARA\&A, 36, 369

Woitke, P. 2006, A\&A, 460, L9

Zeidler, S., Posch, T., \& Mutschke, H. 2013, A\&A, 553, A81

Zhao-Geisler, R., Quirrenbach, A., Köhler, R., Lopez, B., \& Leinert, C. 2011, A\&A, 530, A120 